\title{
Finite-Time Projective Lag Synchronization and Identification between Multiple Weights Markovian Jumping Complex Networks with Stochastic Perturbations
}

\author{
Qian Xie $\mathbb{D}^{1},{ }^{1}$ Changhui Mu $\mathbb{D}^{1},{ }^{1}$ Tong Wang $\mathbb{D}^{2},{ }^{2}$ Gang Wu, ${ }^{3}$ and Rong Jia $\mathbb{D}^{1}$ \\ ${ }^{1}$ Institute of Water Resources and Hydro-electric Engineering, Xi'an University of Technology, Xi'an 710048, China \\ ${ }^{2}$ Shaanxi Provincial Natural Gas Co., Ltd., Shaanxi Gas Group Co., Ltd., Xi'an 710016, China \\ ${ }^{3}$ Changbei Operating Company, PetroChina Changqing Oilfield Company, Xi'an 710018, China
}

Correspondence should be addressed to Qian Xie; xieq@xaut.edu.cn

Received 14 October 2019; Revised 2 February 2020; Accepted 20 February 2020; Published 8 April 2020

Academic Editor: Cornelio Posadas-Castillo

Copyright (c) 2020 Qian Xie et al. This is an open access article distributed under the Creative Commons Attribution License, which permits unrestricted use, distribution, and reproduction in any medium, provided the original work is properly cited.

Two nonidentical dimension Markovian jumping complex networks with stochastic perturbations are taken as objects. The network models under two conditions including single weight and double weights are established, respectively, to study the problem of synchronization and identification. A finite-time projection lag synchronization method is proposed and the unknown parameters of the network are identified. First of all, based on Itô's formula and the stability theory of finite-time, a credible finite-time adaptive controller is presented to guarantee the synchronization of two nonidentical dimension Markovian jumping complex networks with stochastic perturbations under both conditions. Meanwhile, in order to identify the uncertain parameters of the network with stochastic perturbations accurately, some corresponding sufficient conditions are given. Finally, numerical simulations under two working conditions are given to demonstrate the effectiveness and feasibility of the main theory result.

\section{Introduction}

Complex network, a form of system structure and function, is an essential abstraction of the interaction of the complex system and has attracted substantial number of interest from researchers in varied realm $[1,2]$. The World Wide Web, power network, and neural network as well as social network all belong to the complex network of our life. Synchronization is a momentous nonlinear phenomenon of nature. As a most valuable topic in many dynamic behaviors of the complex network, it has attracted a growing number of concerns in the investigation $[3,4]$. If the dynamic behavior of the coupling node in the network evolves over time and eventually reaches the same state, then it will be called synchronization. At present, a series of related research results have been established, including complete synchronization, exponential synchronization, asymptotic synchronization, and generalized synchronization. In [5], a method of complete synchronization of complex networks is proposed by constructing adaptive control technology. A sufficient condition for exponential synchronization of a complex network with time-varying delays is given in [6]. Among these research studies, the dimensions of networks are identical. However, in some practical situations this hypothesis is inappropriate and there are still a large number of networks formed with nonidentical dimensions. Projective synchronization, an important synchronization phenomenon, has received adequate attention which can be used as an appropriate synchronization scheme for the nonidentical dimension complex networks.

Moreover, the abovementioned network synchronization assumes that the synchronization time tends to be infinite $[7,8]$. However, in the engineering field, it is often needed to achieve synchronization as quickly as possible, which means finite-time synchronization. In order to accomplish faster synchronization in complex dynamical 
networks, an effective approach is adopting finite-time control methods. In addition, as an important factor, stochastic perturbations will ineluctably affect the dynamical of the complex network and even destroy the stability of the system in some kinds of practical situations [9-11]. Therefore, in complex dynamical networks, both the stochastic perturbations and how to achieve finite-time synchronization cannot be ignored. Withal, at reality operation conditions, the topology of the network would be randomly switched over time [12,13]. To this end, a Markovian process is introduced to describe. Because of the powerful mathematical modeling ability of the Markovian process in many situations, this kind of network has been developed and studied promptly.

At the meantime, in many complex network synchronization research studies, the system parameters are known. In [14], the asymptotic synchronization of complex networks with nonlinear nodes of different dimensions is studied, but the parameters of the node system are known. However, this situation is not sufficient in the process of practical. The problem of Markovian jumping complex network synchronization with uncertain system parameters needs to be considered.

The research studies on complex networks at home and abroad mainly focus on the synchronization analysis of networks with single weight [15-18]. However, there are few studies on complex networks with multiple weights and seldom involve the synchronization and parameter identification of Markovian jumping complex networks with multiple weights. It is widespread in our life that there are many complex networks with multiple weights which are composed of many subnetworks of different nature, such as communication networks, traffic networks, and interpersonal networks as well as power networks $[19,20]$. Taking the power network as an example, considering each city as a node and the power transmission line between two cities as an edge, there may be a variety of voltage levels of the power transmission network between the two cities, such as highvoltage network and low-voltage network (as shown in Figure 1). Such a power network constitutes a complex network with multiple weights.

Being different from the single weight, its network topology and node dynamics will be more complex. When dealing with multiweighted networks, the method of merging the edges of different properties into one edge for processing is the simplest and most common. However, some edges of the network in real life cannot be simply merged and processed, which cannot truly reflect the characteristics of the network [21].

Inspired by relevant research, this paper discusses the identification and synchronization problem of single-weight [22] and double-weights Markovian jumping complex networks with stochastic perturbations in finite time. This paper will divide double-weights network into two singleweight subnetworks. Then, it is superimposed to establish the network model by the thought of network decomposition. As far as the author knows, there are rarely studies on the identification and synchronization of nonidentical dimension Markovian jumping complex networks with

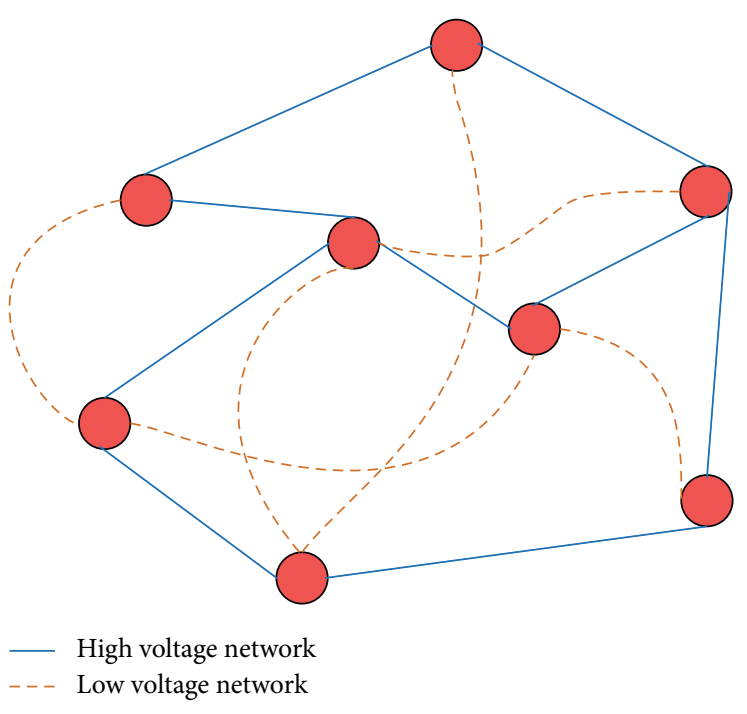

Figure 1: Schematic diagram of the power network.

uncertain parameters in finite time; hence, it is worthwhile to conduct research from the practice and theory.

\section{Preliminaries}

Firstly, some significantly mathematical notations are introduced as follows, which will be applied throughout this paper. Let matrix $A^{T}$ (or $x^{T}$ ) means the transpose of the $A$ (or $x$ ). Denote $\|x\|$ as the 2 -norm. Indicate $I_{n} \in R^{n \times n}$ as the $n$ dimensional identity matrix. Let $\otimes$ represent the Kronecker product of the matrix. $\lambda_{\max }(\cdot)$ is the largest eigenvalue of a matrix.

Definition 1 (see [4]). Define $f: R^{n} \times R_{+} \times \mathcal{S} \longrightarrow R^{n}$ and $g: R^{n} \times R_{+} \times \mathcal{S} \longrightarrow R^{n \times m}$ are differentiable functions satisfying the following $n$-dimensional functional stochastic differential equation:

$$
\mathrm{d} x(t)=f(x(t), t, r(t)) \mathrm{d} t+g(x(t), t, r(t)) \mathrm{d} \omega(t) .
$$

According to Itô's formula, if $V(x(t), t, r) \epsilon$ $C^{2,1}\left(R^{n} \times R_{+} \times \mathcal{S} ; R_{+}\right)$, the operator $\mathscr{L} V$ is defined as follows:

$$
\begin{aligned}
\mathscr{L} V(x(t), t, p)= & V_{t}(x(t), t, p)+V_{x}(x(t), t, p) f(x(t), t, p) \\
& \left.+\frac{1}{2} \operatorname{trance}\left[g^{T}(x(t) t), p\right) V_{x x} g(x(t), t, p)\right] \\
& +\sum_{q=1}^{m} \pi_{p q} V(x(t), t, q),
\end{aligned}
$$

where $V_{t}(x(t), t, p)=(\partial V(x(t), t, p)) / \partial t, \quad V_{x}(x(t), t, p)$ $=\left((\partial V(x(t), t, p)) / \partial x_{1}, \ldots,(\partial V(x(t), t, p)) / \partial x_{n}\right)$, and $V_{x x}$ $(x(t), t, p)=\left(\left(\partial^{2} V(x(t), t, p)\right) /\left(\partial x_{i} \partial x_{j}\right)\right)_{n \times n}$.

Assumption 1. The uniform Lipschitz condition is satisfied by the noise intensity function $\sigma_{i}\left(t, e_{i}(t)\right)$ and the constant $\rho_{i} \geq 0$ is existed to make the following formula hold: 


$$
\operatorname{trance}\left[\sigma_{i}^{T}\left(t, e_{i}(t), r(t)\right) \sigma_{i}\left(t, e_{i}(t), r(t)\right)\right] \leq \rho_{i}(r(t)) e_{i}^{T}(t) e_{i}(t)
$$

Lemma 1 (see [23]). As for any vectors $x, y \in R^{n}$, the following matrix inequality will be hold, where the $P \in R^{n \times n}$ is a positive definite matrix:

$$
2 x^{T} y \leq\left(x^{T} P x+y^{T} P^{-1} y\right) \text {. }
$$

Lemma 2. To suppose that $N(t)$, a continuous, positive definite function, satisfies the following differential inequality $N(t) \leq-\alpha N^{\eta}(t), \forall t \geq t_{0}$, and $N\left(t_{0}\right) \geq 0$, where constants $\alpha>0$ and $0<\eta<1$. As for arbitrary $t_{0}$ and $N(t)$, the following differential inequality will be satisfied:

$$
N^{1-\eta}(t) \leq N^{1-\eta}\left(t_{0}\right)-\alpha(1-\eta)\left(t-t_{0}\right), \quad t_{0} \leq t \leq t_{1},
$$

$N(t) \equiv 0, \forall t \geq t_{1}$. With $t_{1}$, it is given as follows:

$$
t_{1}=t_{0}+\frac{N^{1-\eta}\left(t_{0}\right)}{\alpha(1-\eta)} \text {. }
$$

\section{Lemma 3.}

$$
\left|a_{1}\right|^{\gamma}+\left|a_{2}\right|^{\gamma}+\cdots+\left|a_{n}\right|^{\gamma} \geq\left(\left|a_{1}\right|^{2}+\left|a_{2}\right|^{2}+\cdots+\left|a_{n}\right|^{2}\right)^{\gamma / 2} \text {. }
$$

As well as

$$
\sum_{i=1}^{n}\left|a_{n}\right|^{\gamma} \geq\left(\sum_{i=1}^{n}\left|a_{n}\right|^{2}\right)^{r / 2}
$$

where $\forall \mathrm{a}_{1}, \mathrm{a}_{2}, \ldots, \mathrm{a}_{\mathrm{n}} \in \mathrm{R}^{\mathrm{n}}$ are any vectors and $\gamma$ is a real number satisfying $0<\gamma<2$.

\section{Finite-Time-Generalized Matrix Projective Synchronization with Single- Weight Networks}

The single-weight Markovian jumping complex network with random perturbations is taken as the research object and its parameter identification and finite-time synchronization are studied. The corresponding synchronization criteria and updating rules are obtained as well as the reliability and validity of the method are illustrated by numerical simulation. This is a preparation for further research on synchronization of complex networks with double weights.

3.1. Network Models. The drive-coupled complex network is described as follows, and its number of dynamic nodes is $N$ :

$$
\dot{x}(t)=f_{i 1}\left(x_{i}(t)\right) \alpha_{i}+f_{i 2}\left(x_{i}(t)\right)+\sum_{j=1}^{N} a_{i j}(r(t)) \Gamma_{1} x_{j}(t),
$$

where $x_{i}(t)=\left(x_{i 1}(t), x_{i 2}(t), \ldots, x_{i n}(t)\right)^{T} \in R^{n}$ are the state vectors of the $i$ th node; $f_{i 1}: R^{n} \longrightarrow R^{n \times l}$ is a continuous matrix function and $f_{i 2}: R^{n} \longrightarrow R^{n}$ is a continuous vector function, respectively; $\alpha_{i} \in R^{l}$ are the unidentified node dynamic parameters; $\Gamma_{1} \in R^{n \times n}$ represents the inner coupling matrix; the coupling configuration matrix is expressed by $A(r(t))=\left(a_{i j} r(t)\right)_{N \times N} \in R^{n \times n}$, which represents the topological structure and the coupling strength of the network at time $t$ in mode $r(t)$.

$\{r(t, t \geq 0)\}$ is defined as a right-continuous Markovian process in probability space, describing the switching state between different parameters of time $t$. It takes values from the finite space $\mathcal{S}=\{1,2, \ldots, m\}$ with generator $\Pi=\left(\pi_{p q}\right)_{m \times m}(p, q \in \mathcal{S})$. The transition probability from $p$ th mode at time $t$ to the $q$ th mode at time $t+\Delta t$ will be defined as follows:

$P\{r(t+\Delta t)=q \mid r(t)=p\}= \begin{cases}\pi_{p q} \Delta t+o(\Delta t), & \text { if } q \neq p, \\ 1+\pi_{p q} \Delta t+o(\Delta t), & \text { if } q=p,\end{cases}$

where $\Delta t>0$ :

$$
\lim _{\Delta t \longrightarrow 0} \frac{o(\Delta t)}{\Delta t}=0
$$

As the transition rate $\pi_{p q} \geq 0$ which can be represented from mode $p$ at time $t$ to mode $q$ at time $t+\Delta t$,

$$
\pi_{p p}=-\sum_{q=1, q \neq p}^{m} \pi_{p q} .
$$

Therefore, the elements of matrix $A(r(t))$ will be defined as follows. If it exists as a link from node $j$ to node $i(j \neq i)$, then $a_{i j} \neq 0$; otherwise, $a_{i j}=0$. The diagonal elements of matrix $A(r(t))$ can be determined by $a_{i i}=-\sum_{j=1, j \neq i}^{N} a_{i j}$.

Describe the response-coupled network as follows:

$$
\begin{aligned}
\dot{y}_{i}(t)= & g_{i 1}\left(y_{i}(t)\right) \beta_{i}+g_{i 2}\left(y_{i}(t)\right)+\sum_{j=1}^{N} b_{i j}(r(t)) \Gamma_{2} y_{i}(t) \\
& +u_{i}(t)+\sigma_{i}\left(t, e_{i}(t), r(t)\right) \dot{\omega}_{t},
\end{aligned}
$$

where $y_{i}(t)=\left(y_{i 1}(t), y_{i 2}(t), \ldots, y_{i m}(t)\right)^{T} \in R^{m}$ are the state vectors of node $i ; g_{i}: R^{m} \longrightarrow R^{m \times o}$ is a continuous matrix function and $g_{i_{2}}: R^{m} \longrightarrow R^{m}$ is a continuous vector function; the node dynamic parameter vectors which need to be identified are $\beta_{i} \in R^{o} ; \Gamma_{2} \in R^{m \times m}$ represents the inner coupling matrix; the coupling configuration matrix is defined by $B(r(t))=\left(b_{i j} r(t)\right)_{M \times M} \in R^{m \times m}$ as well as in network (9); $u_{i}(t)$ is the finite-time nonlinear feedback controller which needs to be devised; $\sigma_{i}\left(t, e_{i}(t)\right.$, and $\left.r(t)\right)$ are the noisy intensity function which mainly describe the impact of environmental fluctuations or imprecise design of coupling strength on network synchronization; and $e_{i}(t)$ is the error of system synchronization. follows:

The error of system synchronization can be defined as

$$
e_{i}(t)=y_{i}(t)-M x_{i}(t-\tau)
$$


where $M \in R^{m \times n}$ is the scaling matrix (i.e., generalized matrix) consisting of constant; $e_{i}(t)=\left(e_{i 1}(t), e_{i 2}(t)\right.$, $\left.\ldots, e_{i m}(t)\right)^{T}$; and $\tau$ is the time delay.

Definition 2. The driving network and response network can be realized synchronization through the designed finite-time nonlinear controllers. As for scaling matrix $M_{i j}=\left(m_{i j}\right) \epsilon$ $R^{m \times n}$, if the condition can be satisfied that each row of the element cannot be 0 at a time, it will hold that

$$
\lim _{t \longrightarrow \infty} E\left\|e_{i}(t)\right\|_{2}=\lim _{t \longrightarrow \infty} E\left\|y_{i}(t)-M x_{i}(t-\tau)\right\|_{2}=0 \text {. }
$$

3.2. Main Result. In this section, the synchronization and identification of unknown parameters for Markovian jumping complex networks with nonidentical dimensions and stochastic perturbations are investigated by utilizing the control theory of finite-time. The following theorem and remarks are the main results.

First, from (9) and (13), the synchronization error dynamic system can be obtained as below:

$$
\begin{aligned}
\dot{e}_{i}(t)= & \dot{y}_{i}(t)-M \dot{x}_{i}(t-\tau) \\
= & g_{i 1}\left(y_{i}(t)\right) \beta_{i}+g_{i 2}\left(y_{i}(t)\right)+u_{i}(t)+\sigma_{i}\left(t, e_{i}(t), r(t)\right) \dot{\omega}_{t} \\
& +\sum_{j=1}^{N} b_{i j}(r(t)) \Gamma_{2} e_{i}(t)+\sum_{j=1}^{N} b_{i j}(r(t)) \Gamma_{2} M x_{i}(t-\tau) \\
& -M\left[f_{i 1}\left(x_{i}(t-\tau)\right) \alpha_{i}+f_{i 2}\left(x_{i}(t-\tau)\right)\right] \\
& -M \sum_{j=1}^{N} a_{i j}(r(t)) \Gamma_{1} x_{j}(t-\tau) .
\end{aligned}
$$

Theorem 1. With Assumption 1 holding, the single-weight Markovian jumping complex network (9) and (13) can be realized synchronization in a finite-time. Meanwhile, with the following controllers and undated laws, the value of uncertain parameters can be simultaneously identified:

$$
\begin{aligned}
u_{i}(t)= & -g_{i 1}\left(y_{i}(t)\right) \widehat{\beta}_{i}-g_{i 2}\left(y_{i}(t)\right)+M f_{i 1}\left(x_{i}(t-\tau)\right) \widehat{\alpha}_{i}+M f_{i 2}\left(x_{i}(t-\tau)\right)-k_{i}(r) e_{i}(t) \\
& +M \sum_{j=1}^{N}\left(a_{i j}(r(t)) \Gamma_{1}-b_{i j}(r(t)) \Gamma_{2}\right) x_{j}(t-\tau)-\psi \operatorname{diag}\left(\left|e_{i 1}(t)\right|^{\gamma}, \ldots,\left|e_{i m}(t)\right|^{\gamma}\right) \operatorname{sign}\left(e_{i}(t)\right), \\
\dot{\hat{\alpha}}_{i}(t)= & -\theta_{i}\left[f_{i 1}^{T}\left(x_{i}(t-\tau)\right) M^{T}(t) e_{i}(t)+\frac{\psi}{\theta_{i}}\left(\widehat{\alpha}_{i}-\alpha_{i}\right)^{\gamma} \operatorname{sign}\left(\widehat{\alpha}_{i}-\alpha_{i}\right)\right], \\
\dot{\hat{\beta}}_{i}(t)= & \lambda_{i}\left[g_{i 1}^{T}\left(x_{i}(t)\right) e_{i}(t)-\frac{\psi}{\lambda_{i}}\left(\widehat{\beta}_{i}-\beta_{i}\right)^{\gamma} \operatorname{sign}\left(\widehat{\beta}_{i}-\beta_{i}\right)\right],
\end{aligned}
$$

where $k_{i}(r)>0,0<\gamma<2, \psi>0, \theta_{i}$ and $\lambda_{i}$ are any positive constant, and sign(.) represents symbolic function.

Remark 1. When Markovian jumping complex network (9) and (13) achieve projection lag synchronization in a finite time, the uncertain parameter vectors $\widehat{\alpha}_{i}$ and $\widehat{\beta}_{i}$ of the system are identified.

If the following inequalities hold,

$$
\begin{array}{r}
\sum_{q=1}^{m} \pi_{p q}(D(q)-C(p)) \leq 0, \\
\widehat{B}(r)-(\Xi(r)+\Theta(r)) \otimes I_{m} \leq 0,
\end{array}
$$

where $D(q)$ is a positive definite matrix; $C(p)$ is a symmetrical matrix; $\widehat{B}(r)=\left(\widetilde{B}(r) \widetilde{B}^{T}(r)\right) / 2 ; \widetilde{B}(r)=B(r) \otimes \Gamma_{2}$; $\Xi(r)=\operatorname{diag}\left\{k_{1}(r), k_{2}(r), \ldots, k_{N}(r)\right\} ; \quad \Theta(r)=\operatorname{diag}\left\{\rho_{1}(r)\right.$, $\rho_{2}(r), \ldots, \rho_{N}(r)$; and $r \in S$.
Therefore, the synchronization between single-weight Markovian jumping complex network (9) and (13) with stochastic perturbations can be achieved global asymptotic stability in a finite-time under this condition:

$$
t_{1} \leq t_{0}+\frac{2\left(V\left(t_{0}\right)\right)^{(1-\gamma) / 2}}{\sqrt{2} \psi(1-\gamma)}
$$

where $\quad V\left(t_{0}\right)=(1 / 2) \sum_{i=1}^{N} e_{i}^{T}\left(t_{0}\right) e_{i}\left(t_{0}\right)+(1 / 2) \sum_{i=1}^{N}\left(1 / \theta_{i}\right)$ $\widetilde{\alpha}_{i}^{T} \widetilde{\alpha}_{i}+(1 / 2) \sum_{i=1}^{N}\left(1 / \lambda_{i}\right) \widetilde{\beta}_{i}^{T} \widetilde{\beta}_{i}$.

Proof. The Lyapunov function is defined as below:

$$
V(t, e(t), p)=\frac{1}{2} \sum_{i=1}^{N} e_{i}^{T}(t) e_{i}(t)+\frac{1}{2} \sum_{i=1}^{N} \frac{1}{\theta_{i}} \widetilde{\alpha}_{i}^{T} \widetilde{\alpha}_{i}+\frac{1}{2} \sum_{i=1}^{N} \frac{1}{\lambda_{i}} \widetilde{\beta}_{i}^{T} \widetilde{\beta}_{i} .
$$

By Definition 1, we can obtain the differential operator $\mathscr{L} \mathrm{V}$ as follows: 


$$
\begin{aligned}
\mathscr{L} V(x(t), t, p)= & \sum_{i=1}^{N} e_{i}^{T}(t) \dot{e}_{i}(t)+\sum_{i=1}^{N} \frac{1}{\theta_{i}} \stackrel{\dot{\hat{\alpha}}_{i}}{\tilde{\alpha}_{i}}+\sum_{i=1}^{N} \frac{1}{\lambda_{i}} \stackrel{\dot{\hat{\beta}}_{i}}{\widetilde{\beta}_{i}}+\sum_{q=1}^{m} \pi_{p q} \sum_{i=1}^{N} e_{i}^{T}(t) D(q) e(t) \\
& +\sum_{i=1}^{N} \operatorname{trance}\left[\sigma_{i}^{T}\left(t, e_{i}(t), p\right) \sigma_{i}\left(t, e_{i}(t), p\right)\right] .
\end{aligned}
$$

Substitute (16) and feedback controllers (17) into (21), resulting in

$$
\begin{aligned}
\mathscr{L} V(x(t), t, p)= & \sum_{i=1}^{N} e_{i}^{T}(t)\left\{g_{i 1}\left(y_{i}(t)\right) \beta_{i}+\sigma_{i}\left(t, e_{i}(t), r(t)\right) \dot{\omega}_{t}+\sum_{j=1}^{N} b_{i j}(r(t)) \Gamma_{2} e_{i}(t)+\sum_{j=1}^{N} b_{i j}(r(t)) \Gamma_{2} M x_{i}(t-\tau)+u_{i}(t)\right. \\
& \left.+g_{i 2}\left(y_{i}(t)\right)-M\left[f_{i 1}\left(x_{i}(t-\tau)\right) \alpha_{i}+f_{i 2}\left(x_{i}(t-\tau)\right)\right]-M \sum_{j=1}^{N} a_{i j}(r(t)) \Gamma_{1} x_{j}(t-\tau)\right\} \\
& -\sum_{i=1}^{N} e_{i}^{T}(t) M f_{i 1}\left(x_{i}(t-\tau)\right) \widetilde{\alpha}_{i}+\sum_{i=1}^{N} e_{i}^{T}(t) g_{i 1}\left(x_{i}(t)\right) \widetilde{\beta}_{i}-\psi \sum_{i=1}^{N} \frac{1}{\theta_{i}} \widetilde{\alpha}_{i}\left(\widehat{\alpha}_{i}-\alpha_{i}\right)^{\gamma} \operatorname{sign}\left(\widehat{\alpha}_{i}-\alpha_{i}\right) \\
& -\psi \sum_{i=1}^{N} \frac{1}{\lambda_{i}} \widetilde{\beta}_{i}\left(\widehat{\beta}_{i}-\beta_{i}\right)^{\gamma} \operatorname{sign}\left(\widehat{\beta}_{i}-\beta_{i}\right)+\sum_{i=1}^{N} \operatorname{trance}\left[\sigma_{i}^{T}\left(t, e_{i}(t), p\right) \sigma_{i}\left(t, e_{i}(t), p\right)\right]+\sum_{q=1}^{m} \pi_{p q} \sum_{i=1}^{N} e_{i}^{T}(t) D(q) e(t), \\
u_{i}(t)= & -g_{i 1}\left(y_{i}(t)\right) \widehat{\beta}_{i}-g_{i 2}\left(y_{i}(t)\right)+M f_{i 1}\left(x_{i}(t-\tau)\right) \widehat{\alpha}_{i}+M f_{i 2}\left(x_{i}(t-\tau)\right)-k_{i}(r) e_{i}(t) \\
& +M \sum_{j=1}^{N}\left(a_{i j}(r(t)) \Gamma_{1}-b_{i j}(r(t)) \Gamma_{2}\right) x_{j}(t-\tau)-\psi \operatorname{diag}\left(\left|e_{i 1}(t)\right|^{\gamma}, \ldots,\left|e_{i m}(t)\right|^{\gamma}\right) \operatorname{sign}\left(e_{i}(t)\right),
\end{aligned}
$$

$$
\begin{aligned}
\mathscr{L} V(x(t), t, p)= & \sum_{i=1}^{N} e_{i}^{T}(t)\left\{g_{i 1}\left(y_{i}(t)\right) \beta_{i}+\sigma_{i}\left(t, e_{i}(t), r(t)\right) \dot{\omega}_{t}+\sum_{j=1}^{N} b_{i j}(r(t)) \Gamma_{2} e_{i}(t)+\sum_{j=1}^{N} b_{i j}(r(t)) \Gamma_{2} M x_{i}(t-\tau)\right. \\
& +g_{i 2}\left(y_{i}(t)\right)-M\left[f_{i 1}\left(x_{i}(t-\tau)\right) \alpha_{i}+f_{i 2}\left(x_{i}(t-\tau)\right)\right]-M \sum_{j=1}^{N} a_{i j}(r(t)) \Gamma_{1} x_{j}(t-\tau)-g_{i 1}\left(y_{i}(t)\right) \widehat{\beta}_{i} \\
& -g_{i 2}\left(y_{i}(t)\right)+M f_{i 1}\left(x_{i}(t-\tau)\right) \widehat{\alpha}_{i}+M f_{i 2}\left(x_{i}(t-\tau)\right)-k_{i}(r) e_{i}(t)+M \sum_{j=1}^{N}\left(a_{i j}(r(t)) \Gamma_{1}-b_{i j}(r(t)) \Gamma_{2}\right) x_{j}(t-\tau) \\
& \left.-\psi \operatorname{diag}\left(\left|e_{i 1}(t)\right|^{\gamma}, \ldots,\left|e_{i m}(t)\right|^{\gamma}\right) \operatorname{sign}\left(e_{i}(t)\right)\right\}, \\
& -\sum_{i=1}^{N} e_{i}^{T}(t) M f_{i 1}\left(x_{i}(t-\tau)\right) \widetilde{\alpha}_{i}+\sum_{i=1}^{N} e_{i}^{T}(t) g_{i 1}\left(x_{i}(t)\right) \widetilde{\beta}_{i}-\psi \sum_{i=1}^{N} \frac{1}{\theta_{i}} \widetilde{\alpha}_{i}\left(\widehat{\alpha}_{i}-\alpha_{i}\right)^{\gamma} \operatorname{sign}\left(\widehat{\alpha}_{i}-\alpha_{i}\right) \\
& -\psi \sum_{i=1}^{N} \frac{1}{\lambda_{i}} \tilde{\beta}_{i}\left(\widehat{\beta}_{i}-\beta_{i}\right)^{\gamma} \operatorname{sign}\left(\widehat{\beta}_{i}-\beta_{i}\right)+\sum_{i=1}^{N} \operatorname{trance}\left[\sigma_{i}^{T}\left(t, e_{i}(t), p\right) \sigma_{i}\left(t, e_{i}(t), p\right)\right]+\sum_{q=1}^{m} \pi_{p q} \sum_{i=1}^{N} e_{i}^{T}(t) D(q) e(t),
\end{aligned}
$$




$$
\begin{aligned}
& \mathscr{L} V(x(t), t, p)=\sum_{i=1}^{N} e_{i}^{T}(t)\left\{M f_{i 1}\left(x_{i}(t-\tau)\right)\left(\widehat{\alpha}_{i}(t)-\alpha_{i}\right)-g_{i 1}\left(y_{i}(t)\right)\left(\widehat{\beta}_{i}(t)-\beta_{i}\right)+\sum_{j=1}^{N} b_{i j}(r(t)) \Gamma_{2} e_{i}(t)-k_{i}(r) e_{i}(t)\right. \\
& -\psi \operatorname{diag}\left(\left|e_{i 1}(t)\right|^{\gamma}, \ldots,\left|e_{i m}(t)\right|^{\gamma}\right) \operatorname{sign}\left(e_{i}(t)\right\} \\
& -\sum_{i=1}^{N} e_{i}^{T}(t) M f_{i 1}\left(x_{i}(t-\tau)\right) \widetilde{\alpha}_{i}+\sum_{i=1}^{N} e_{i}^{T}(t) g_{i 1}\left(y_{i}(t)\right) \widetilde{\beta}_{i}-\psi \sum_{i=1}^{N} \frac{1}{\theta_{i}} \widetilde{\alpha}_{i}\left(\widehat{\alpha}_{i}-\alpha_{i}\right)^{\gamma} \operatorname{sign}\left(\widehat{\alpha}_{i}-\alpha_{i}\right) \\
& -\psi \sum_{i=1}^{N} \frac{1}{\lambda_{i}} \tilde{\beta}_{i}\left(\widehat{\beta}_{i}-\beta_{i}\right)^{\gamma} \operatorname{sign}\left(\widehat{\beta}_{i}-\beta_{i}\right)+\sum_{i=1}^{N} \operatorname{trance}\left[\sigma_{i}^{T}\left(t, e_{i}(t), p\right) \sigma_{i}\left(t, e_{i}(t), p\right)\right] \\
& +\sum_{q=1}^{m} \pi_{p q} \sum_{i=1}^{N} e_{i}^{T}(t) D(q) e(t) \\
& \mathscr{L} V(x(t), t, p)=\sum_{i=1}^{N} e_{i}^{T}(t)\left\{\sum_{j=1}^{N} b_{i j}(r(t)) \Gamma_{2} e_{i}(t)-k_{i}(r) e_{i}(t)-\psi \operatorname{diag}\left(\left|e_{i 1}(t)\right|^{\gamma}, \ldots,\left|e_{i m}(t)\right|^{\gamma}\right) \operatorname{sign}\left(e_{i}(t)\right)\right\} \\
& +\sum_{i=1}^{N} \operatorname{trance}\left[\sigma_{i}^{T}\left(t, e_{i}(t), p\right) \sigma_{i}\left(t, e_{i}(t), p\right)\right]-\psi \sum_{i=1}^{N} \frac{1}{\theta_{i}} \widetilde{\alpha}_{i}\left(\widehat{\alpha}_{i}-\alpha_{i}\right)^{\gamma} \operatorname{sign}\left(\widehat{\alpha}_{i}-\alpha_{i}\right) \\
& -\psi \sum_{i=1}^{N} \frac{1}{\lambda_{i}} \widetilde{\beta}_{i}\left(\widehat{\beta}_{i}-\beta_{i}\right)^{\gamma} \operatorname{sign}\left(\widehat{\beta}_{i}-\beta_{i}\right)+\sum_{q=1}^{m} \pi_{p q} \sum_{i=1}^{N} e_{i}^{T}(t) D(q) e(t) .
\end{aligned}
$$

According to Lemma 3, we obtain that

$$
\begin{aligned}
\sum_{i=1}^{N} e_{i}^{T}(t)\left|e_{i}(t)\right|^{\varphi} \operatorname{sign}\left(e_{i}(t)\right) & =\sum_{i=1}^{N} e_{i}^{T}(t) \operatorname{diag}\left(\left|e_{i 1}(t)\right|^{\gamma}, \ldots,\left|e_{i m}(t)\right|^{\gamma}\right) \operatorname{sign}\left(e_{i}(t)\right) \\
& =\sum_{i=1}^{N} \sum_{j=1}^{N} e_{i j}(t)\left|e_{i j}(t)\right|^{\gamma} \operatorname{sign}\left(e_{i j}(t)\right)=\sum_{i=1}^{N} \sum_{j=1}^{N}\left|e_{i j}(t)\right|^{1+\gamma} \\
& \geq\left(\sum_{i=1}^{N} \sum_{j=1}^{N}\left|e_{i j}(t)\right|^{2}\right)^{(1+\gamma) / 2}=\left(\sum_{i=1}^{N} e_{i}^{T}(t) e_{i}(t)\right)^{(1+\gamma) / 2} \\
& \Longrightarrow \sum_{i=1}^{N} e_{i}^{T}(t)\left|e_{i}(t)\right|^{\varphi} \operatorname{sign}\left(e_{i}(t)\right) \geq\left(\sum_{i=1}^{N} e_{i}^{T}(t) e_{i}(t)\right)^{(1+\gamma) / 2} .
\end{aligned}
$$

From Assumption 1, the conclusion is drawn:

$$
\begin{aligned}
\mathscr{L} V(x(t), t, p) \leq & \sum_{i=1}^{N} e_{i}^{T}(t)\left(\widehat{B}(r)-(\Xi(r)+\Theta(r)) \otimes I_{m}\right) e_{i}(t)+\sum_{q=1}^{m} \pi_{p q} \sum_{i=1}^{N} e_{i}^{T}(t)(D(q)-C(p)) e(t)-\psi\left(\sum_{i=1}^{N} e_{i}^{T}(t) e_{i}(t)\right){ }^{(1+\gamma) / 2} \\
& -\psi\left(\sum_{i=1}^{N} \frac{1}{\theta_{i}} \widetilde{\alpha}_{i}^{T} \widetilde{\alpha}_{i}\right)^{(1+\gamma) / 2}-\psi\left(\sum_{i=1}^{N} \frac{1}{\lambda_{i}} \widetilde{\beta}_{i}^{T} \widetilde{\beta}_{i}\right)^{(1+\gamma) / 2} \leq-\psi\left(\sum_{i=1}^{N} e_{i}^{T}(t) e_{i}(t)+\sum_{i=1}^{N} \frac{1}{\theta_{i}} \widetilde{\alpha}_{i}^{T} \widetilde{\alpha}_{i}+\sum_{i=1}^{N} \frac{1}{\lambda_{i}} \widetilde{\beta}_{i}^{T} \widetilde{\beta}_{i}\right)^{(1+\gamma) / 2} \\
\leq & -\sqrt{2} \psi\left(\frac{1}{2} \sum_{i=1}^{N} e_{i}^{T}(t) e_{i}(t)+\frac{1}{2} \sum_{i=1}^{N} \frac{1}{\theta_{i}} \widetilde{\alpha}_{i}^{T} \widetilde{\alpha}_{i}+\frac{1}{2} \sum_{i=1}^{N} \frac{1}{\lambda_{i}} \widetilde{\beta}_{i}^{T} \widetilde{\beta}_{i}\right)^{(1+\gamma) / 2}=-\sqrt{2} \psi(V(t))^{(1+\gamma) / 2} \\
\Longrightarrow & \mathscr{L} V(x(t), t, p) \leq-\sqrt{2} \psi(V(t))^{(1+\gamma) / 2} .
\end{aligned}
$$


From Lemma 2, the system error of synchronization $e_{i}(t)$ will converge to a steady state with a finite-time, which can be estimated by

$$
\begin{aligned}
& t_{1} \leq t_{0}+\frac{\left(V\left(t_{0}\right)\right)^{1-((1+\gamma) / 2)}}{\sqrt{2} \psi(1-((1+\gamma) / 2))}=t_{0}+\frac{2\left(V\left(t_{0}\right)\right)^{(1-\gamma) / 2}}{\sqrt{2} \psi(1-\gamma)} \\
& \Longrightarrow t_{1} \leq t_{0}+\frac{2\left(V\left(t_{0}\right)\right)^{(1-\gamma) / 2}}{\sqrt{2} \psi(1-\gamma)},
\end{aligned}
$$

where $\quad V\left(t_{0}\right)=(1 / 2) \sum_{i=1}^{N} e_{i}^{T}\left(t_{0}\right) e_{i}\left(t_{0}\right)+(1 / 2) \sum_{i=1}^{N}\left(1 / \theta_{i}\right)$ $\widetilde{\alpha}_{i}^{T} \widetilde{\alpha}_{i}+(1 / 2) \sum_{i=1}^{N}\left(1 / \lambda_{i}\right) \widetilde{\beta}_{i}^{T} \widetilde{\beta}_{i}$.

Hence, networks (9) and (13) can be realized synchronization with finite time $t_{1}$. Also, the uncertain system parameter vectors $\widehat{\alpha}_{i}$ and $\widehat{\beta}_{i}$ are adapted to the true value of parameters. The proof is completed.

Remark 2. The proposed approach is applicable to the finitetime-generalized matrix projection lag synchronization of any two single-weight Markovian jumping complex networks with uncertain parameters, stochastic perturbations, and different initial conditions. In addition, the Markovian jumping complex network can be identical or nonidentical dimensions.

Remark 3. The synchronization and identification speed can be adjusted through choosing the constants $k_{i}(r), \theta_{i}(r)$, and $\lambda_{i}(r)$, adequately.

Remark 4. Inequality (18) in Theorem 1 is only just sufficient but not a necessary condition for single-weight Markovian jumping complex networks (9) and (13) to realize projection lag synchronization and parameter identification.

3.3. Simulation Results. Some numerical simulations are performed to prove the feasibility and effectiveness of the abovementioned result. The Chen system is taken as the node dynamic of the drive complex network:

$$
\left(\begin{array}{c}
\dot{x}_{i 1}(t) \\
\dot{x}_{i 2}(t) \\
\dot{x}_{i 3}(t)
\end{array}\right)=\left(\begin{array}{c}
0 \\
-x_{i 1}(t) x_{i 3}(t) \\
x_{i 1}(t) x_{i 2}(t)
\end{array}\right)+\left(\begin{array}{ccc}
x_{i 2}(t)-x_{i 1}(t) & 0 & 0 \\
-x_{i 1}(t) & 0 & x_{i 1}(t)+x_{i 2}(t) \\
0 & -x_{i 3}(t) & 0
\end{array}\right)\left(\begin{array}{c}
\alpha_{i 1} \\
\alpha_{i 2} \\
\alpha_{i 3}
\end{array}\right) .
$$

The identifying value of the parameter vector $\alpha_{i}$ is $\alpha_{i}=\left(\alpha_{i 1}, \alpha_{i 2}, \alpha_{i 3}\right)^{T}=(35,3,28)^{T}$.
The four-dimensional hyperchaotic Lüchaotic system will be the node dynamic of the response complex network:

$$
\left(\begin{array}{c}
\dot{x}_{i 1}(t) \\
\dot{x}_{i 2}(t) \\
\dot{x}_{i 3}(t) \\
\dot{x}_{i 4}(t)
\end{array}\right)=\left(\begin{array}{c}
0 \\
-x_{i 1}(t) x_{i 3}(t) \\
x_{i 1}(t) x_{i 2}(t) \\
x_{i 1}(t) x_{i 3}(t)
\end{array}\right)+\left(\begin{array}{cccc}
x_{i 2}(t)-x_{i 1}(t) & 0 & 0 & x_{i 4}(t) \\
0 & 0 & \left.x_{i 2}(t)\right) & 0 \\
0 & -x_{i 3}(t) & 0 & 0 \\
0 & 0 & 0 & x_{i 4}(t)
\end{array}\right)\left(\begin{array}{c}
\beta_{i 1} \\
\beta_{i 2} \\
\beta_{i 3} \\
\beta_{i 4}
\end{array}\right) .
$$

And the identifying value of the parameter vector $\beta_{i}$ is $\beta_{i}=\left(\beta_{1}, \beta_{2}, \beta_{3}, \beta_{4}\right)^{T}=(36,3,20,1)^{T}$.

The size of network is taken as $N=10$. The coupling configuration matrices of the network are separately given, as shown in Figures 2 and 3. Figure 2 is the topological structure of the driving system with mode 1 and mode 2 . Figure 3 is the response system. Among them, the structure of network topology changing with two modes is shown with the dotted line.
In order to judge the quality of the network synchronization, we define the system error value as follows:

$$
E(t)=\sqrt{\sum_{i=1}^{N} \sum_{j=1}^{N} \frac{e_{i j}^{2}(t)}{N}}
$$

When $\lim _{t} E(t)=0$ and $\forall t>t_{1}$, systems (9) and (13) will implement global synchronization within the finite time $t_{1}$. 
The other relevant parameters are as below:

$$
\begin{aligned}
& \Gamma_{1}=\left[\begin{array}{ccc}
0.1 & 0 & 0 \\
0 & 0.1 & 0 \\
0 & 0 & 0.1
\end{array}\right] \text {, } \\
& \Gamma_{2}=\left[\begin{array}{cccc}
0.2 & 0 & 0 & 0 \\
0 & 0.2 & 0 & 0 \\
0 & 0 & 0.2 & 0 \\
0 & 0 & 0 & 0.2
\end{array}\right], \\
& M=\left[\begin{array}{ccc}
0.2 & 0 & -0.1 \\
0.1 & -0.5 & 5.5 \\
-2 & -1 & 3 \\
-0.5 & 1.2 & -2
\end{array}\right], \\
& \sigma_{i}\left(t, e_{i}(t), 1\right)=\operatorname{diag}\left\{\left(\frac{\sqrt{2}}{2}\right) e_{i 1}(t),\left(\frac{\sqrt{2}}{2}\right) e_{i 1}(t),\left(\frac{\sqrt{2}}{2}\right) e_{i 1}(t)\right\}, \\
& \sigma_{i}\left(t, e_{i}(t), 2\right)=\operatorname{diag}\left\{\sqrt{2} e_{i 1}(t), \sqrt{2} e_{i 1}(t), \sqrt{2} e_{i 1}(t)\right\} \text {. }
\end{aligned}
$$

The switching of two different modes in the complex system can be shown at Figure 4. $\widehat{\alpha}_{i}(0) \in R^{3 \times 1}$ and $\widehat{\beta}_{i}(0) \in R^{4 \times 1}$ are selected arbitrary integer in the interval $[0,1]$ and $\tau=0.01$. The parameters of controller (17) are chosen as follows: $k_{i}(1)=k_{i}(2)=15, \quad \gamma=0.6$, and $\lambda_{i}(1)=\lambda_{i}(2)=12$. Through Lemma 2 and (28), we can obtain $t_{1}=7.4729$ by simple calculation.

The time-varying curves of the system synchronization errors $e_{i}(t)(1 \leq i \leq 4)$ are demonstrated in Figure 5. From that, one can see that the error converge to zero which means that the two networks are in the synchronization state. Figure 6 is the system error values $E(t)$. The conclusion of Figure 6 matches that of Figure 5. Figures 7 and 8 give the identification of parameters $\widehat{\alpha}_{i}(i=1,2,3)$ and $\widehat{\beta}_{i}(i=1,2,3,4)$. It is clear that the estimated parameters are approached to the value $(35,3,28)^{T}$ and $(36$, $3,20,1)^{T}$. They all attest that the control scheme can realize the identification and synchronization of two single-weight Markonian jumping complex networks, effectively.

\section{Finite-Time-Generalized Matrix Projective Synchronization with Double- Weight Networks}

In order to build a practical Markovian jumping complex network model with double-weights, this paper introduces a method of network splitting, which divides the different coupling configuration into different subnetworks. Each network after splitting has its own nature and structure. In this paper, different side voltage levels in the power network are defined as different properties, which are divided into two subnetworks (as shown in Figure 9). Then, the finitetime generalized function lag projection synchronization is studied. The synchronization criteria are given and the values of the related unknown parameters are identified further.

4.1. Network Models. In this section, the driving system is defined as follows and its dynamic node number is $N$ :

$$
\begin{aligned}
x(t)= & f_{i 1}\left(x_{i}(t)\right) \alpha_{i}+f_{i 2}\left(x_{i}(t)\right)+\sum_{j=1}^{N} a_{i j}(r(t)) \Gamma_{1} x_{j}(t) \\
& +\sum_{j=1}^{N} b_{i j}(r(t)) \Gamma_{2} x_{j}(t),
\end{aligned}
$$

where $\Gamma_{i}(i=1,2)$ are the inner coupling matrices; $A(r(t))=\left(a_{i j} r(t)\right)_{N \times N}$ and $B(r(t))=\left(b_{i j} r(t)\right)_{N \times N}$ represent the coupling configuration matrices which are represented as different coupling relationships. Its elements satisfy the following. If there exists a link from 


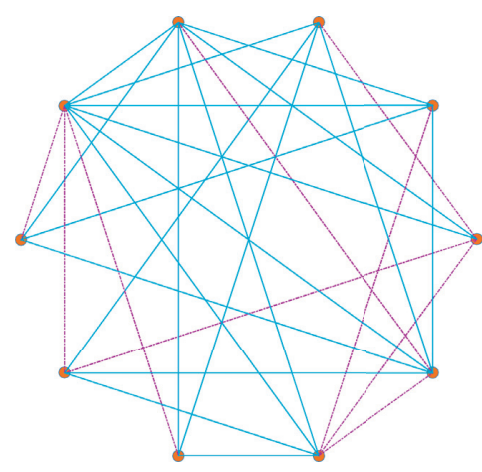

(a)

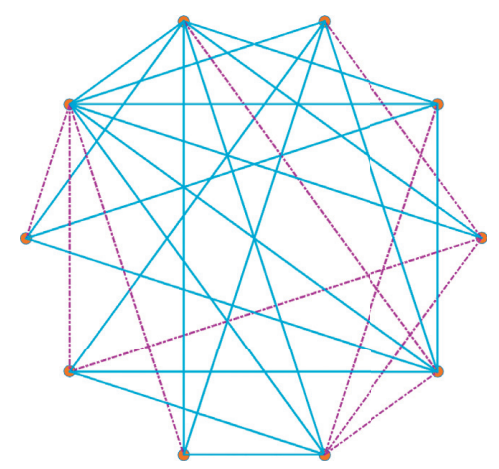

(b)

FIgURE 2: (a) Topological structure of the driving system in mode 1 and (b) topological structure of the driving system in mode 2.

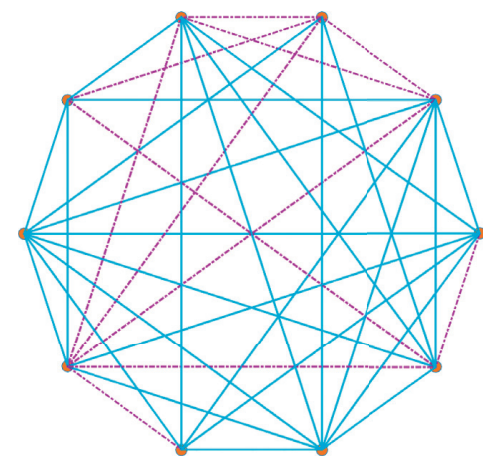

(a)

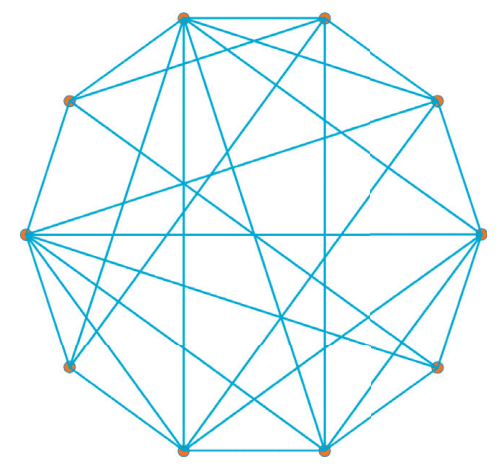

(b)

Figure 3: (a) Topological structure of the response system in mode 1 and (b) topological structure of the response system in mode 2.

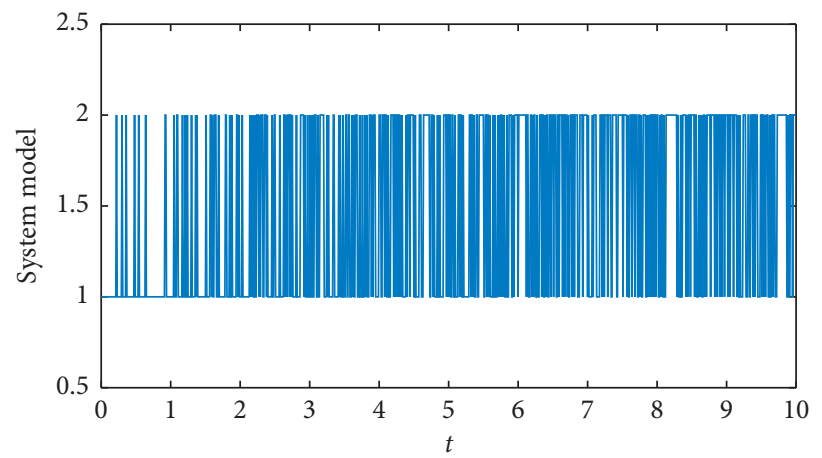

FIGURE 4: Switching of the system mode.

node $j$ to node $i(j \neq i)$, then $a_{i j} \neq 0\left(b_{i j} \neq 0\right)$; otherwise, $a_{i j}=0\left(b_{i j}=0\right)$. The diagonal elements of matrix $A(r(t))(B(r(t)))$ are defined as

$$
a_{i i}=-\sum_{j=1, j \neq i}^{N} a_{i j}\left(b_{i i}=-\sum_{j=1, j \neq i}^{N} b_{i j}\right) .
$$

The response coupled network can be described as follows:

$$
\begin{aligned}
\dot{y}_{i}(t)= & g_{i 1}\left(y_{i}(t)\right) \beta_{i}+g_{i 2}\left(y_{i}(t)\right)+\sum_{j=1}^{N} m_{i j}(r(t)) \Gamma_{3} y_{i}(t) \\
& +\sum_{j=1}^{N} n_{i j}(r(t)) \Gamma_{4} y_{i}(t)+u_{i}(t)+\sigma_{i}\left(t, e_{i}(t), r(t)\right) \dot{\omega}_{t},
\end{aligned}
$$

where $\Gamma_{i}(i=3,4)$ represent the inner coupling matrices; $M(r(t))=\left(m_{i j} r(t)\right)_{N \times N}$ and $N(r(t))=\left(n_{i j} r(t)\right)_{N \times N}$ are 


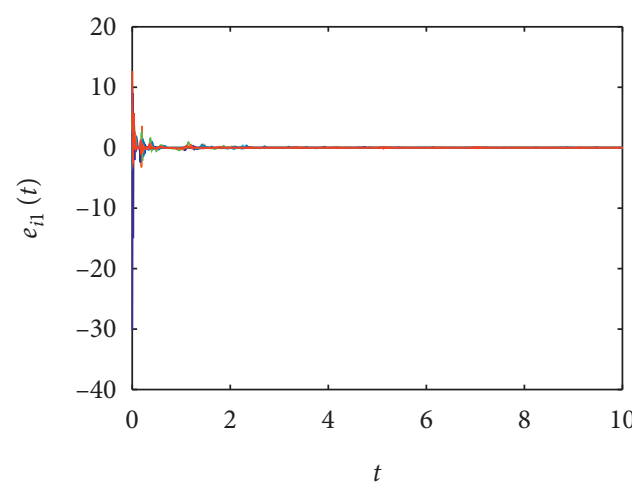

(a)

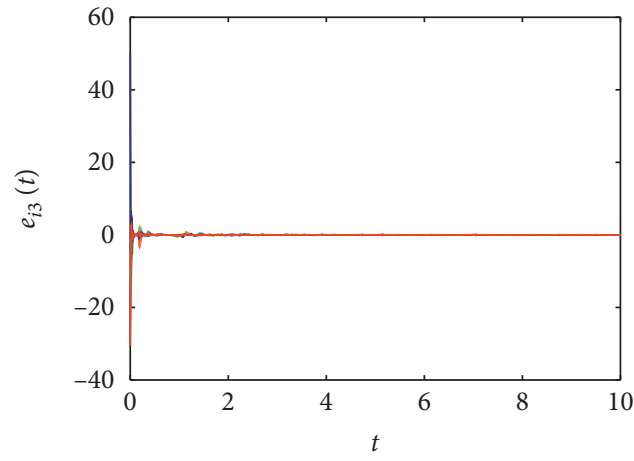

(c)

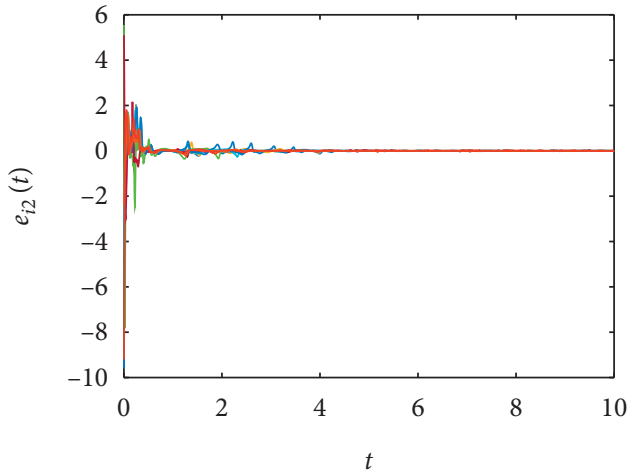

(b)

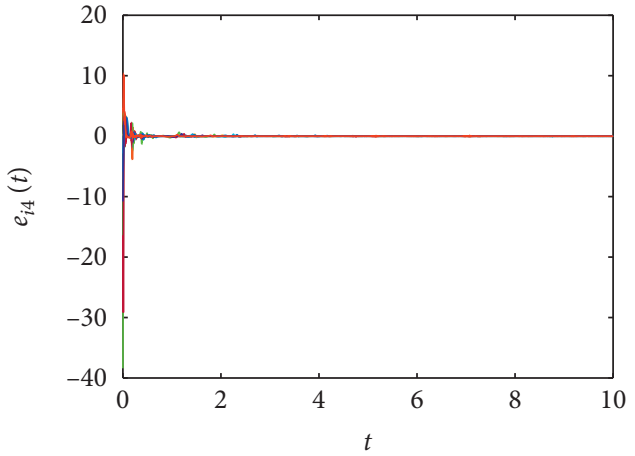

(d)

FIGURE 5: Synchronization errors $e_{i}(t)$ with time evolution.

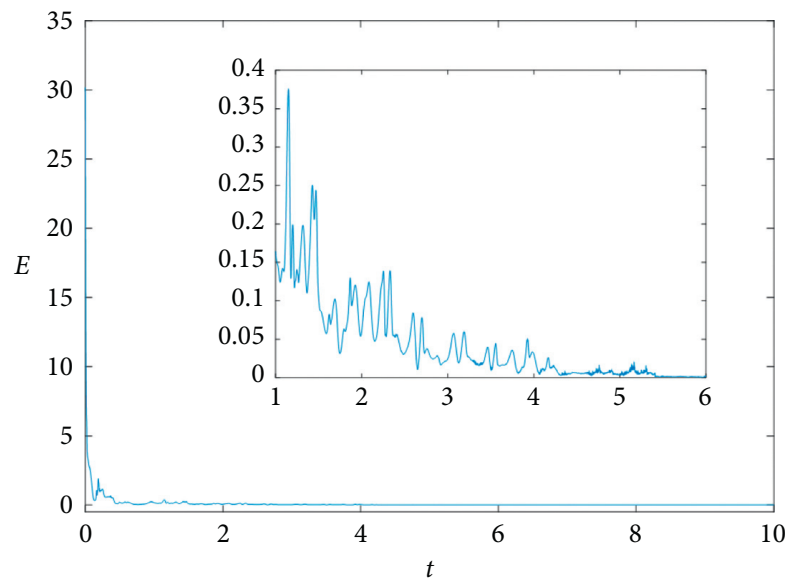

FIGURE 6: Synchronization error value $E(t)$ with varying time.

the coupling configuration matrices, same as $A(r(t))(B(r(t)))$ in $(33) ; u_{i}(t)$ is the finite-time nonlinear feedback controller which needs to be designed; and $\sigma_{i}\left(t, e_{i}(t), r(t)\right)$ is the noisy intensity function and $e_{i}(t)$ is the synchronization error.

The system synchronization error is defined as follows:

$$
e_{i}(t)=y_{i}(t)-M(t) x_{i}(t-\tau),
$$

where $M(t) \in R^{m \times n}$ is the scaling matrix consisting of functions (i.e., generalized function matrix).
Definition 3. The driving network (33) and response network (35) can be realized synchronization with the designed finite-time nonlinear controllers. As for scaling matrix $M(t)=\left(m_{i j}(t)\right) \in R^{m \times n}$, if the condition can be satisfied that the each row of element cannot be 0 at a time, it will hold that

$$
\lim _{t \longrightarrow \infty} E\left\|e_{i}(t)\right\|_{2}=\lim _{t \longrightarrow \infty} E\left\|y_{i}(t)-M(t) x_{i}(t-\tau)\right\|_{2}=0 .
$$




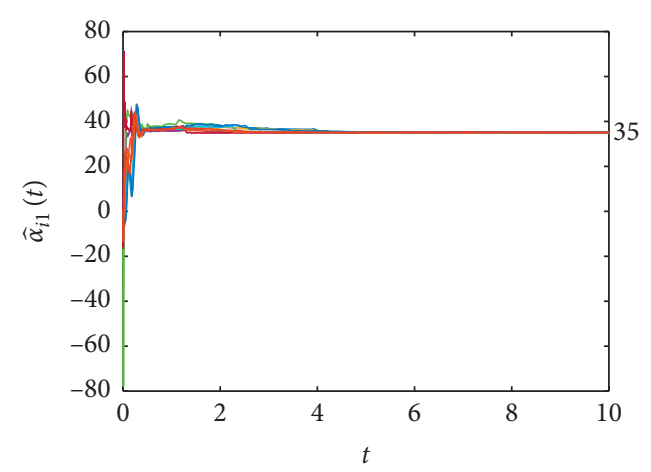

(a)

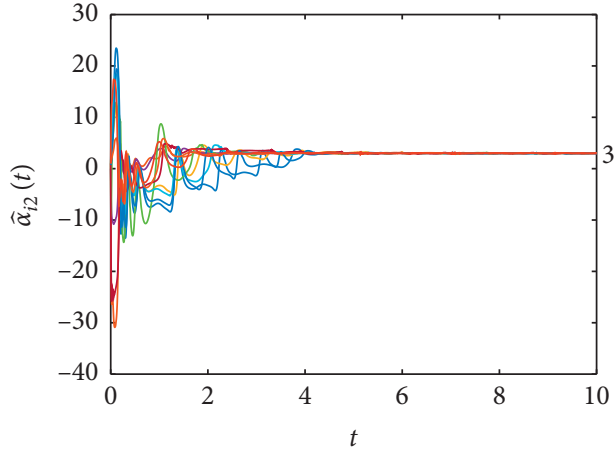

(b)

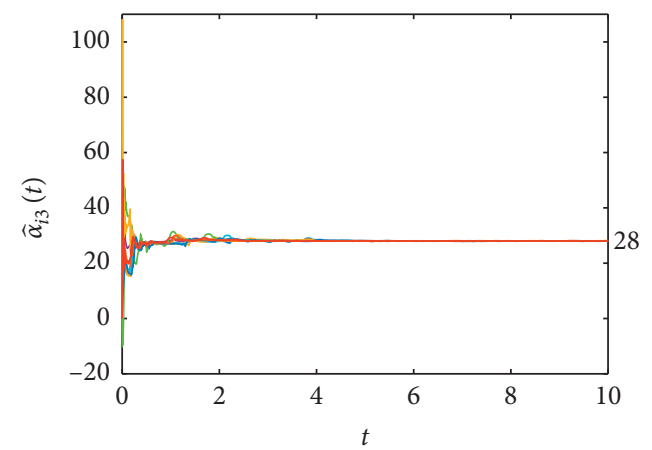

(c)

FIGURE 7: Identification of the uncertain parameter $\widehat{\alpha}_{i}(t)$.

4.2. Main Result. The synchronization error of the dynamic system is obtained as the following from (33) and (35):

$$
\begin{aligned}
\dot{e}_{i}(t)= & \dot{y}_{i}(t)-M \dot{x}_{i}(t-\tau)-\dot{M} x_{i}(t-\tau) \\
= & g_{i 1}\left(y_{i}(t)\right) \beta_{i}+g_{i 2}\left(y_{i}(t)\right)+u_{i}(t)+\sigma_{i}\left(t, e_{i}(t), r(t)\right) \dot{\omega}_{t}+\sum_{j=1}^{N} m_{i j}(r(t)) \Gamma_{3} e_{i}(t) \\
& +\sum_{j=1}^{N} m_{i j}(r(t)) \Gamma_{3} M(t) x_{i}(t-\tau)+\sum_{j=1}^{N} n_{i j}(r(t)) \Gamma_{4} e_{i}(t)-\dot{M}(t) x_{i}(t-\tau) \\
& +\sum_{j=1}^{N} n_{i j}(r(t)) \Gamma_{4} M(t) x_{i}(t-\tau)-M(t)\left[f_{i 1}\left(x_{i}(t-\tau)\right) \alpha_{i}+f_{i 2}\left(x_{i}(t-\tau)\right)\right] \\
& -M(t) \sum_{j=1}^{N} a_{i j}(r(t)) \Gamma_{1} x_{j}(t-\tau)-M(t) \sum_{j=1}^{N} b_{i j}(r(t)) \Gamma_{2} x_{j}(t-\tau) .
\end{aligned}
$$

Theorem 2. With Assumption 1 holding, the doubleweights Markovian jumping complex networks (33) and (35) can be realized synchronization in a finite-time.
Meanwhile, with following controllers and undated laws, the value of uncertain network parameters can be simultaneously identified: 
12

Complexity

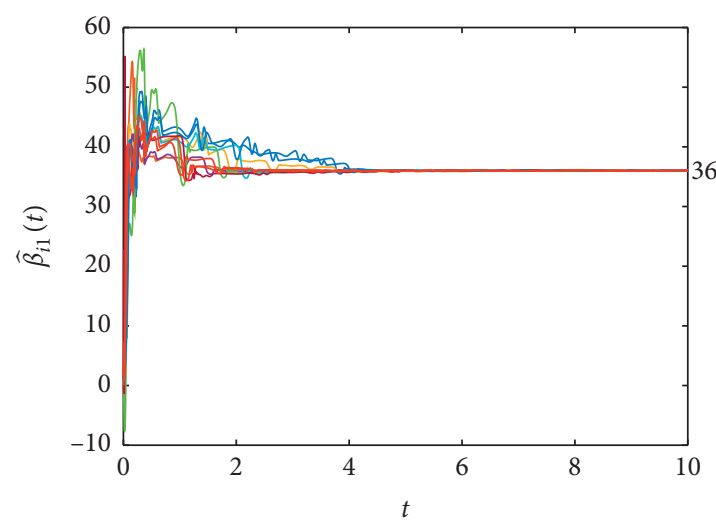

(a)

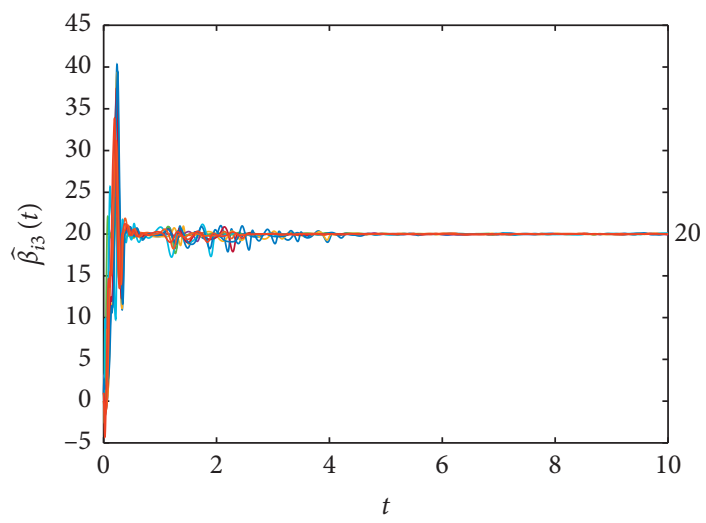

(c)

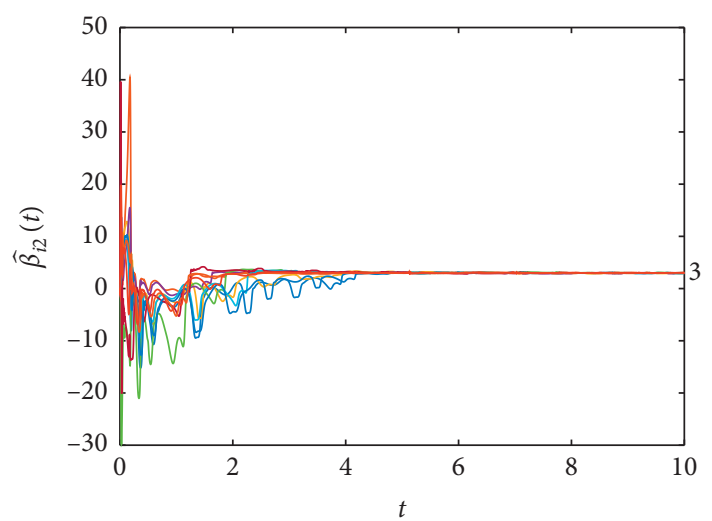

(b)

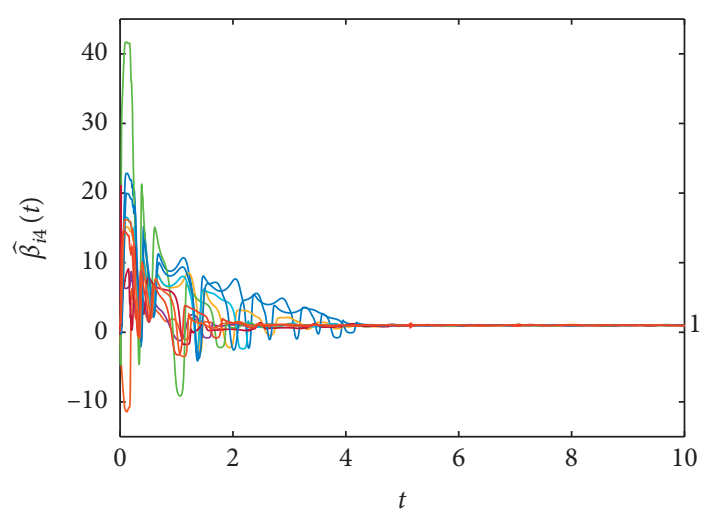

(d)

FIgURE 8: Identification of the uncertain parameter $\widehat{\beta}_{i}(t)$.

$$
\begin{aligned}
u_{i}(t)= & \dot{M}(t) x_{i}(t-\tau)-g_{i 1}\left(y_{i}(t)\right) \widehat{\beta}_{i}-g_{i 2}\left(y_{i}(t)\right)+M f_{i 1}\left(x_{i}(t-\tau)\right) \widehat{\alpha}_{i}+M f_{i 2}\left(x_{i}(t-\tau)\right) \\
& -k_{i}(r) e_{i}(t)+\sum_{j=1}^{N}\left(a_{i j}(r(t)) M(t) \Gamma_{1}-m_{i j}(r(t)) M(t) \Gamma_{3}\right) x_{j}(t-\tau) \\
& +\sum_{j=1}^{N}\left(b_{i j}(r(t)) M(t) \Gamma_{2}-n_{i j}(r(t)) M(t) \Gamma_{4}\right) x_{j}(t-\tau) \\
& -\psi \operatorname{diag}\left(\left|e_{i 1}(t)\right|^{\gamma}, \ldots,\left|e_{i m}(t)\right|^{\gamma}\right) \operatorname{sign}\left(e_{i}(t)\right), \\
\dot{\hat{\alpha}}_{i}(t)= & -h_{i}(r)\left[f_{i 1}^{T}\left(x_{i}(t-\tau)\right) M^{T}(t) e_{i}(t)+\frac{\psi}{h_{i}(r)}\left(\widehat{\alpha}_{i}-\alpha_{i}\right)^{\gamma} \operatorname{sign}\left(\widehat{\alpha}_{i}-\alpha_{i}\right)\right] \\
\dot{\hat{\beta}}_{i}(t)= & s_{i}(r)\left[g_{i 1}^{T}\left(x_{i}(t)\right) e_{i}(t)-\frac{\psi}{s_{i}(r)}\left(\widehat{\beta}_{i}-\beta_{i}\right)^{\gamma} \operatorname{sign}\left(\widehat{\beta}_{i}-\beta_{i}\right)\right] .
\end{aligned}
$$




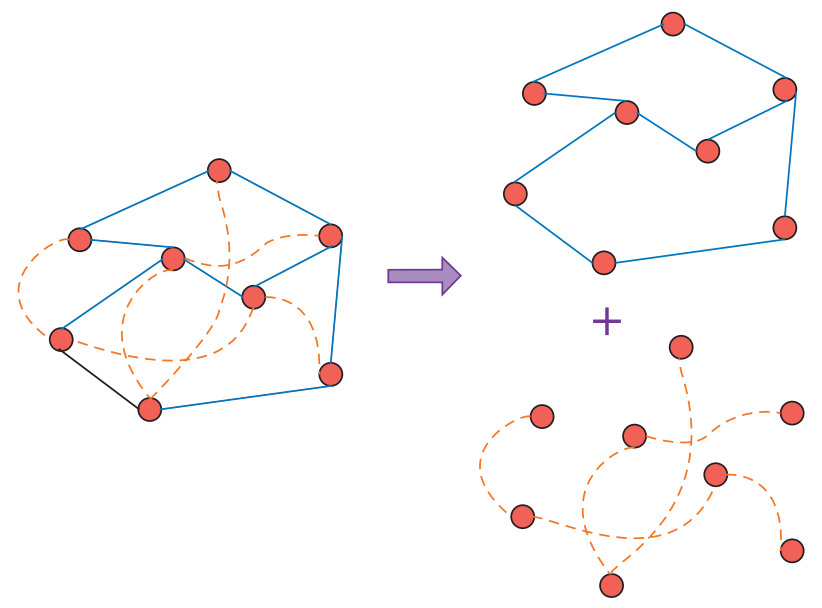

Figure 9: Network splitting diagram.

Remark 5. When Markovian jumping complex networks (33) and (35) achieve projection lag synchronization in a finite time, the uncertain system parameter vectors $\widehat{\alpha}_{i}$ and $\widehat{\beta}_{i}$ are identified.

If the following inequalities hold,

$$
\begin{array}{r}
\sum_{q=1}^{m} \pi_{p q}(D(q)-C(p)) \leq 0, \\
\widetilde{Q}(r)+\widetilde{P}(r)+(\Theta(r)-\Xi(r)) \otimes I_{m} \leq 0,
\end{array}
$$

where $D(q)$ is a positive definite matrix; $C(p)$ is a symmetrical matrix; $\widetilde{Q}(r)=Q(r) \otimes \Gamma_{3} ; \widetilde{P}(r)=P(r) \otimes \Gamma_{4} ; \Theta(r)=$ $\operatorname{diag}\left\{\rho_{1}(r), \rho_{2}(r), \ldots, \rho_{N}(r) ; \quad \Xi(r)=\operatorname{diag}\left\{k_{1}(r), k_{2}(r), \ldots\right.\right.$, $\left.k_{N}(r)\right\}$; and $r \in S$.

Therefore, the synchronization between double-weights Markovian jumping complex networks (33) and (35) with stochastic perturbations can be achieved global asymptotic stability in a finite-time under this condition:

$$
t_{1} \leq t_{0}+\frac{2\left(V\left(t_{0}\right)\right)^{(1-\gamma) / 2}}{\sqrt{2} \psi(1-\gamma)},
$$

where $V\left(t_{0}\right)=(1 / 2) \sum_{i=1}^{N} e_{i}^{T}\left(t_{0}\right) e_{i}\left(t_{0}\right)+(1 / 2) \sum_{i=1}^{N}\left(1 / h_{i}(r)\right)$ $\widetilde{\alpha}_{i}^{T} \widetilde{\alpha}_{i}+(1 / 2) \sum_{i=1}^{N}\left(1 / s_{i}(r)\right) \widetilde{\beta}_{i}^{T} \widetilde{\beta}_{i}$.

Proof. The Lyapunov function is defined as follows:

$$
\begin{aligned}
V(t, e(t), p)= & \frac{1}{2} \sum_{i=1}^{N} e_{i}^{T}(t) e_{i}(t)+\frac{1}{2} \sum_{i=1}^{N} \frac{1}{h_{i}(p)} \widetilde{\alpha}_{i}^{T} \widetilde{\alpha}_{i} \\
& +\frac{1}{2} \sum_{i=1}^{N} \frac{1}{s_{i}(p)} \widetilde{\beta}_{i}^{T} \widetilde{\beta}_{i} .
\end{aligned}
$$

By Definition 1, the differential operator $\mathscr{L V}$ is given as

$$
\begin{aligned}
\mathscr{L} V(x(t), t, p)= & \sum_{i=1}^{N} e_{i}^{T}(t) \dot{e}_{i}(t)+\sum_{i=1}^{N} \frac{1}{h_{i}(p)} \dot{\hat{\alpha}}_{i}^{T} \widetilde{\alpha}_{i}+\sum_{i=1}^{N} \frac{1}{s_{i}(p)} \dot{\hat{\beta}}_{i}^{T} \widetilde{\beta}_{i} \\
& +\sum_{q=1}^{m} \pi_{p q} \sum_{i=1}^{N} e_{i}^{T}(t) D(q) e(t)+\sum_{i=1}^{N} \operatorname{trance}\left[\sigma_{i}^{T}\left(t, e_{i}(t), p\right) \sigma_{i}\left(t, e_{i}(t), p\right)\right] .
\end{aligned}
$$

Substitute (38) and feedback controllers (39) into (43), resulting in 


$$
\begin{aligned}
& \mathscr{L} V(x(t), t, p)=\sum_{i=1}^{N} e_{i}^{T}(t)\left\{g_{i 1}\left(y_{i}(t)\right) \beta_{i}+g_{i 2}\left(y_{i}(t)\right)+u_{i}(t)+\sigma_{i}\left(t, e_{i}(t), r(t)\right) \dot{\omega}_{t}+\sum_{j=1}^{N} m_{i j}(r(t)) \Gamma_{3} e_{i}(t)\right. \\
& +\sum_{j=1}^{N} m_{i j}(r(t)) \Gamma_{3} M(t) x_{i}(t-\tau)+\sum_{j=1}^{N} n_{i j}(r(t)) \Gamma_{4} e_{i}(t)+\sum_{j=1}^{N} n_{i j}(r(t)) \Gamma_{4} M(t) x_{i}(t-\tau) \\
& \left.-M(t)\left[f_{i 1}\left(x_{i}(t-\tau)\right) \alpha_{i}+f_{i 2}\left(x_{i}(t-\tau)\right)\right]-M(t) \sum_{j=1}^{N} a_{i j}(r(t)) \Gamma_{1} x_{j}(t-\tau)-M(t) \sum_{j=1}^{N} b_{i j}(r(t)) \Gamma_{2} x_{j}(t-\tau)\right\} \\
& -\sum_{i=1}^{N} e_{i}^{T}(t) M f_{i 1}\left(x_{i}(t-\tau)\right) \widetilde{\alpha}_{i}+\sum_{i=1}^{N} e_{i}^{T}(t) g_{i 1}\left(x_{i}(t)\right) \widetilde{\beta}_{i} \\
& -\psi \sum_{i=1}^{N} \frac{1}{h_{i}(r)} \widetilde{\alpha}_{i}\left(\widehat{\alpha}_{i}-\alpha_{i}\right)^{\gamma} \operatorname{sign}\left(\widehat{\alpha}_{i}-\alpha_{i}\right)-\psi \sum_{i=1}^{N} \frac{1}{s_{i}(r)} \widetilde{\beta}_{i}\left(\widehat{\beta}_{i}-\beta_{i}\right)^{\gamma} \operatorname{sign}\left(\widehat{\beta}_{i}-\beta_{i}\right) \\
& +\sum_{i=1}^{N} \operatorname{trance}\left[\sigma_{i}^{T}\left(t, e_{i}(t), p\right) \sigma_{i}\left(t, e_{i}(t), p\right)\right]+\sum_{q=1}^{m} \pi_{p q} \sum_{i=1}^{N} e_{i}^{T}(t) D(q) e(t) \\
& u_{i}(t)=\dot{M}(t) x_{i}(t-\tau)-g_{i 1}\left(y_{i}(t)\right) \widehat{\beta}_{i}-g_{i 2}\left(y_{i}(t)\right)+M f_{i 1}\left(x_{i}(t-\tau)\right) \widehat{\alpha}_{i}+M f_{i 2}\left(x_{i}(t-\tau)\right) \\
& -k_{i}(r) e_{i}(t)+\sum_{j=1}^{N}\left(a_{i j}(r(t)) M(t) \Gamma_{1}-m_{i j}(r(t)) M(t) \Gamma_{3}\right) x_{j}(t-\tau) \\
& +\sum_{j=1}^{N}\left(b_{i j}(r(t)) M(t) \Gamma_{2}-n_{i j}(r(t)) M(t) \Gamma_{4}\right) x_{j}(t-\tau) \\
& -\psi \operatorname{diag}\left(\left|e_{i 1}(t)\right|^{\gamma}, \ldots,\left|e_{i m}(t)\right|^{\gamma}\right) \operatorname{sign}\left(e_{i}(t)\right),
\end{aligned}
$$

$$
\begin{aligned}
& \mathscr{L} V(x(t), t, p)=\sum_{i=1}^{N} e_{i}^{T}(t)\left\{g_{i 1}\left(y_{i}(t)\right) \beta_{i}+g_{i 2}\left(y_{i}(t)\right)+\sigma_{i}\left(t, e_{i}(t), r(t)\right) \dot{\omega}_{t}+\sum_{j=1}^{N} m_{i j}(r(t)) \Gamma_{3} e_{i}(t) \sum_{j=1}^{N} m_{i j}(r(t)) \Gamma_{3} M(t) x_{i}(t-\tau)\right. \\
& +\sum_{j=1}^{N} n_{i j}(r(t)) \Gamma_{4} e_{i}\left((t)+\sum_{j=1}^{N} n_{i j}(r(t)) \Gamma_{4} M(t) x_{i}(t-\tau)-M(t)\left[f_{i 1}\left(x_{i}(t-\tau)\right) \alpha_{i}+f_{i 2}\left(x_{i}(t-\tau)\right)\right]\right. \\
& -M(t) \sum_{j=1}^{N} a_{i j}(r(t)) \Gamma_{1} x_{j}(t-\tau)-M(t) \sum_{j=1}^{N} b_{i j}(r(t)) \Gamma_{2} x_{j}(t-\tau) \dot{M}(t) x_{i}(t-\tau)-g_{i 1}\left(y_{i}(t)\right) \widehat{\beta}_{i}-g_{i 2}\left(y_{i}(t)\right) \\
& +M f_{i 1}\left(x_{i}(t-\tau)\right) \widehat{\alpha}_{i}+M f_{i 2}\left(x_{i}(t-\tau)\right)-k_{i}(r) e_{i}(t)+\sum_{j=1}^{N}\left(a_{i j}(r(t)) M(t) \Gamma_{1}-m_{i j}(r(t)) M(t) \Gamma_{3}\right) x_{j}(t-\tau) \\
& \left.+\sum_{j=1}^{N}\left(b_{i j}(r(t)) M(t) \Gamma_{2}-n_{i j}(r(t)) M(t) \Gamma_{4}\right)-x_{j}(t-\tau)-\psi \operatorname{diag}\left(\left|e_{i 1}(t)\right|^{\gamma}, \ldots,\left|e_{i m}(t)\right|^{\gamma}\right) \operatorname{sign}\left(e_{i}(t)\right)\right\} \\
& -\sum_{i=1}^{N} e_{i}^{T}(t) M f_{i 1}\left(x_{i}(t-\tau)\right) \widetilde{\alpha}_{i}+\sum_{i=1}^{N} e_{i}^{T}(t) g_{i 1}\left(x_{i}(t)\right) \widetilde{\beta}_{i} \\
& -\psi \sum_{i=1}^{N} \frac{1}{h_{i}(r)} \widetilde{\alpha}_{i}\left(\widehat{\alpha}_{i}-\alpha_{i}\right)^{\gamma} \operatorname{sign}\left(\widehat{\alpha}_{i}-\alpha_{i}\right)-\psi \sum_{i=1}^{N} \frac{1}{s_{i}(r)} \widetilde{\beta}_{i}\left(\widehat{\beta}_{i}-\beta_{i}\right)^{\gamma} \operatorname{sign}\left(\widehat{\beta}_{i}-\beta_{i}\right) \\
& +\sum_{i=1}^{N} \operatorname{trance}\left[\sigma_{i}^{T}\left(t, e_{i}(t), p\right) \sigma_{i}\left(t, e_{i}(t), p\right)\right]+\sum_{q=1}^{m} \pi_{p q} \sum_{i=1}^{N} e_{i}^{T}(t) D(q) e(t) \text {, }
\end{aligned}
$$




$$
\begin{aligned}
\mathscr{L} V(x(t), t, p)= & \sum_{i=1}^{N} e_{i}^{T}(t)\left\{M(t) f_{i 1}\left(x_{i}(t-\tau)\right)\left(\widehat{\alpha}_{i}(t)-\alpha_{i}\right)-g_{i 1}\left(y_{i}(t)\right)\left(\widehat{\beta}_{i}(t)-\beta_{i}\right)-k_{i}(r) e_{i}(t)\right. \\
& +\sum_{j=1}^{N}\left(m_{i j}(r(t)) \Gamma_{3}+n_{i j}(r(t)) \Gamma_{4}\right) e_{i}(t)-\psi \operatorname{diag}\left(\left|e_{i 1}(t)\right|^{\gamma}, \ldots,\left|e_{i m}(t)\right|^{\gamma}\right) \operatorname{sign}\left(e_{i}(t)\right\} \\
& -\sum_{i=1}^{N} e_{i}^{T}(t) M(t) f_{i 1}\left(x_{i}(t-\tau)\right) \widetilde{\alpha}_{i}+\sum_{i=1}^{N} e_{i}^{T}(t) g_{i 1}\left(y_{i}(t)\right) \widetilde{\beta}_{i} \\
& -\psi \sum_{i=1}^{N} \frac{1}{h_{i}(r)} \widetilde{\alpha}_{i}\left(\widehat{\alpha}_{i}-\alpha_{i}\right)^{\gamma} \operatorname{sign}\left(\widehat{\alpha}_{i}-\alpha_{i}\right)-\psi \sum_{i=1}^{N} \frac{1}{s_{i}(r)} \widetilde{\beta}_{i}\left(\widehat{\beta}_{i}-\beta_{i}\right)^{\gamma} \operatorname{sign}\left(\widehat{\beta}_{i}-\beta_{i}\right) \\
& +\sum_{i=1}^{N} \operatorname{trance}\left[\sigma_{i}^{T}\left(t, e_{i}(t), p\right) \sigma_{i}\left(t, e_{i}(t), p\right)\right]+\sum_{q=1}^{m} \pi_{p q} \sum_{i=1}^{N} e_{i}^{T}(t) D(q) e(t), \\
\mathscr{L} V(x(t), t, p)= & \sum_{i=1}^{N} e_{i}^{T}(t) M(t) f_{i 1}\left(x_{i}(t-\tau)\right)\left(\widehat{\alpha}_{i}(t)-\alpha_{i}\right)-\sum_{i=1}^{N} e_{i}^{T}(t) g_{i 1}\left(y_{i}(t)\right)\left(\widehat{\beta}_{i}(t)-\beta_{i}\right) \\
& +\sum_{i=1}^{N} e_{i}^{T}(t)\left\{-k_{i}(r) e_{i}(t)+\sum_{j=1}^{N}\left(m_{i j}(r(t)) \Gamma_{3}+n_{i j}(r(t)) \Gamma_{4}\right) e_{i}(t)-\psi \operatorname{diag}\left(\left|e_{i 1}(t)\right|^{\gamma}, \ldots,\left|e_{i m}(t)\right|^{\gamma}\right) \operatorname{sign}\left(e_{i}(t)\right\}\right. \\
& -\sum_{i=1}^{N} e_{i}^{T}(t) M(t) f_{i 1}\left(x_{i}(t-\tau)\right) \widetilde{\alpha}_{i}+\sum_{i=1}^{N} e_{i}^{T}(t) g_{i 1}\left(y_{i}(t)\right) \widetilde{\beta}_{i}-\psi \sum_{i=1}^{N} \frac{1}{h_{i}(r)} \widetilde{\alpha}_{i}\left(\widehat{\alpha}_{i}-\alpha_{i}\right)^{\gamma} \operatorname{sign}\left(\widehat{\alpha}_{i}-\alpha_{i}\right) \\
& -\psi \sum_{i=1}^{N} \frac{1}{s_{i}(r)} \widetilde{\beta}_{i}\left(\widehat{\beta}_{i}-\beta_{i}\right)^{\gamma} \operatorname{sign}\left(\widehat{\beta}_{i}-\beta_{i}\right) \\
& +\sum_{i=1}^{N} \operatorname{trance}\left[\sigma_{i}^{T}\left(t, e_{i}(t), p\right) \sigma_{i}\left(t, e_{i}(t), p\right)\right]+\sum_{q=1}^{m} \pi_{p q} \sum_{i=1}^{N} e_{i}^{T}(t) D(q) e(t), \\
&
\end{aligned}
$$

$$
\begin{aligned}
\mathscr{L} V(x(t), t, p)= & \sum_{i=1}^{N} e_{i}^{T}(t)\left\{\sum_{j=1}^{N} m_{i j}(r(t)) \Gamma_{3} e_{i}(t)+\sum_{j=1}^{N} n_{i j}(r(t)) \Gamma_{4} e_{i}(t)-k_{i}(r) e_{i}(t)-\psi \operatorname{diag}\left(\left|e_{i 1}(t)\right|^{\gamma}, \ldots,\left|e_{i m}(t)\right|^{\gamma}\right) \operatorname{sign}\left(e_{i}(t)\right)\right\} \\
& +\sum_{i=1}^{N} \operatorname{trance}\left[\sigma_{i}^{T}\left(t, e_{i}(t), p\right) \sigma_{i}\left(t, e_{i}(t), p\right)\right]-\psi \sum_{i=1}^{N} \frac{1}{h_{i}(r)} \widetilde{\alpha}_{i}\left(\widehat{\alpha}_{i}-\alpha_{i}\right)^{\gamma} \operatorname{sign}\left(\widehat{\alpha}_{i}-\alpha\right)_{i} \\
& -\psi \sum_{i=1}^{N} \frac{1}{s_{i}(r)} \widetilde{\beta}_{i}\left(\widehat{\beta}_{i}-\beta_{i}\right)^{\gamma} \operatorname{sign}\left(\widehat{\beta}_{i}-\beta_{i}\right)+\sum_{q=1}^{m} \pi_{p q} \sum_{i=1}^{N} e_{i}^{T}(t) D(q) e(t) .
\end{aligned}
$$

According to Lemma 3,

$$
\begin{aligned}
\sum_{i=1}^{N} e_{i}^{T}(t)\left|e_{i}(t)\right|^{\varphi} \operatorname{sign}\left(e_{i}(t)\right) & =\sum_{i=1}^{N} e_{i}^{T}(t) \operatorname{diag}\left(\left|e_{i 1}(t)\right|^{\gamma}, \ldots,\left|e_{i m}(t)\right|^{\gamma}\right) \operatorname{sign}\left(e_{i}(t)\right) \\
& =\sum_{i=1}^{N} \sum_{j=1}^{N} e_{i j}(t)\left|e_{i j}(t)\right|^{\gamma} \operatorname{sign}\left(e_{i j}(t)\right)=\sum_{i=1}^{N} \sum_{j=1}^{N}\left|e_{i j}(t)\right|^{1+\gamma} \\
& \geq\left(\sum_{i=1}^{N} \sum_{j=1}^{N}\left|e_{i j}(t)\right|^{2}\right)^{(1+\gamma) / 2}=\left(\sum_{i=1}^{N} e_{i}^{T}(t) e_{i}(t)\right)^{(1+\gamma) / 2} \\
& \Longrightarrow \sum_{i=1}^{N} e_{i}^{T}(t)\left|e_{i}(t)\right|^{\varphi} \operatorname{sign}\left(e_{i}(t)\right) \geq\left(\sum_{i=1}^{N} e_{i}^{T}(t) e_{i}(t)\right)^{(1+\gamma) / 2} .
\end{aligned}
$$


From Assumption 1, the conclusion is drawn:

$$
\begin{aligned}
\mathscr{L} V(x(t), t, p) \leq & e^{T}(t)\left(\widetilde{Q}(r)+\widetilde{P}(r)+(\Theta(r)-\Xi(r)) \otimes I_{m}\right) e(t)+\sum_{q=1}^{m} \pi_{p q} \sum_{i=1}^{N} e_{i}^{T}(t)(D(q)-C(p)) e(t) \\
& -\psi\left(\sum_{i=1}^{N} e_{i}^{T}(t) e_{i}(t)\right)^{(1+\gamma) / 2}-\psi\left(\sum_{i=1}^{N} \frac{1}{h_{i}(r)} \widetilde{\alpha}_{i}^{T} \widetilde{\alpha}_{i}\right)^{(1+\gamma) / 2}-\psi\left(\sum_{i=1}^{N} \frac{1}{s_{i}(r)} \widetilde{\beta}_{i}^{T} \widetilde{\beta}_{i}\right)^{(1+\gamma) / 2} \\
\leq & -\psi\left(\sum_{i=1}^{N} e_{i}^{T}(t) e_{i}(t)+\sum_{i=1}^{N} \frac{1}{\theta_{i}} \widetilde{\alpha}_{i}^{T} \widetilde{\alpha}_{i}+\sum_{i=1}^{N} \frac{1}{\lambda_{i}} \widetilde{\beta}_{i}^{T} \widetilde{\beta}_{i}\right)^{(1+\gamma) / 2} \\
\leq & -\sqrt{2} \psi\left(\frac{1}{2} \sum_{i=1}^{N} e_{i}^{T}(t) e_{i}(t)+\frac{1}{2} \sum_{i=1}^{N} \frac{1}{\theta_{i}} \widetilde{\alpha}_{i}^{T} \widetilde{\alpha}_{i}+\frac{1}{2} \sum_{i=1}^{N} \frac{1}{\lambda_{i}} \widetilde{\beta}_{i}^{T} \widetilde{\beta}_{i}\right)^{(1+\gamma) / 2}=-\sqrt{2} \psi(V(t))^{(1+\gamma) / 2} \\
\Longrightarrow & \mathscr{L} V(x(t), t, p) \leq-\sqrt{2} \psi(V(t))^{(1+\gamma) / 2} .
\end{aligned}
$$

From Lemma 2, the system error of synchronization $e_{i}(t)$ will converge to a steady state with a finite-time which can be estimated by

$$
\begin{gathered}
t_{1} \leq t_{0}+\frac{\left(V\left(t_{0}\right)\right)^{1-((1+\gamma) / 2)}}{\sqrt{2} \psi(1-((1+\gamma) / 2))}=t_{0}+\frac{2\left(V\left(t_{0}\right)\right)^{(1-\gamma) / 2}}{\sqrt{2} \psi(1-\gamma)} \\
\Longrightarrow t_{1} \leq t_{0}+\frac{2\left(V\left(t_{0}\right)\right)^{(1-\gamma) / 2}}{\sqrt{2} \psi(1-\gamma)},
\end{gathered}
$$

where $\quad V\left(t_{0}\right)=1 / 2 \sum_{i=1}^{N} e_{i}^{T}\left(t_{0}\right) e_{i}\left(t_{0}\right)+1 / 2 \sum_{i=1}^{N} 1 /\left(h_{i}(r)\right)$ $\widetilde{\alpha}_{i}^{T} \widetilde{\alpha}_{i}+1 / 2 \sum_{i=1}^{N} 1 /\left(s_{i}(r)\right) \widetilde{\beta}_{i}^{T} \widetilde{\beta}_{i}$.

Hence, networks (33) and (35) could be realized synchronization with finite time $t_{1}$. As well as, the unknown parameters vector $\widehat{\alpha}_{i}$ and $\widehat{\beta}_{i}$ of the system are adapted to the true value with themselves. The proof is completed.

Remark 6. The proposed approach is applicable to the finitetime-generalized matrix projection lag synchronization of any two double-weights Markovian jumping complex networks with uncertain parameters, stochastic perturbations, and different initial conditions. In addition, the dimensions of the Markovian jumping complex network can be identical or nonidentical.

Remark 7. The synchronization and identification speed can be adjusted by choosing constants $k_{i}(r), h_{i}(r)$, and $s_{i}(r)$, adequately.

Remark 8. Inequality (40) in Theorem 2 is only just the sufficient but not necessary condition for double-weights Markovian jumping complex networks (33) and (35) to realize projection lag synchronization and parameter identification.

\subsection{Simulation Results}

4.3.1. Simulation Analysis I. The Chen system is taken as the node dynamic of drive complex network:

$$
\left(\begin{array}{c}
\dot{x}_{i 1}(t) \\
\dot{x}_{i 2}(t) \\
\dot{x}_{i 3}(t)
\end{array}\right)=\left(\begin{array}{c}
0 \\
-x_{i 1}(t) x_{i 3}(t) \\
x_{i 1}(t) x_{i 2}(t)
\end{array}\right)+\left(\begin{array}{ccc}
x_{i 2}(t)-x_{i 1}(t) & 0 & 0 \\
-x_{i 1}(t) & 0 & x_{i 1}(t)+x_{i 2}(t) \\
0 & -x_{i 3}(t) & 0
\end{array}\right)\left(\begin{array}{c}
\alpha_{i 1} \\
\alpha_{i 2} \\
\alpha_{i 3}
\end{array}\right)
$$


The identifying value of the parameter vector $\alpha_{i}$ is $\alpha_{i}=\left(\alpha_{i 1}, \alpha_{i 2}, \alpha_{i 3}\right)^{T}=(35,3,28)^{T}$.
The four-dimensional Lüchaotic system will be the node dynamic of the response complex network:

$$
\left(\begin{array}{c}
\dot{x}_{i 1}(t) \\
\dot{x}_{i 2}(t) \\
\dot{x}_{i 3}(t) \\
\dot{x}_{i 4}(t)
\end{array}\right)=\left(\begin{array}{c}
0 \\
-x_{i 1}(t) x_{i 3}(t) \\
x_{i 1}(t) x_{i 2}(t) \\
x_{i 1}(t) x_{i 3}(t)
\end{array}\right)+\left(\begin{array}{cccc}
x_{i 2}(t)-x_{i 1}(t) & 0 & 0 & x_{i 4}(t) \\
0 & 0 & x_{i 2}(t) & 0 \\
0 & -x_{i 3}(t) & 0 & 0 \\
0 & 0 & 0 & \left.x_{i 4}(t)\right)
\end{array}\right)\left(\begin{array}{c}
\beta_{i 1} \\
\beta_{i 2} \\
\beta_{i 3} \\
\beta_{i 4}
\end{array}\right) .
$$

The identifying value of the parameter vector $\beta_{i}$ is $\beta_{i}=\left(\beta_{1}, \beta_{2}, \beta_{3}, \beta_{4}\right)^{T}=(36,3,20,1)^{T}$.

The size of network is taken as $N=10$. And the coupling configuration matrices of the network are separately given as Figures 10 and 11. Figure 10 is the topological structure of the driving system with mode 1 and mode 2 . And Figure 11 is the response systems. The complex coupling network is divided into two simple coupling structures according to the theory of network splitting. Among the two subgraphs, the structure of network topology change with two modes is shown with the dotted line. The switching of the system mode is shown in Figure 12.

The other relevant parameters are as below:

$$
\begin{aligned}
& \Gamma_{1}=\left[\begin{array}{lll}
1 & 0 & 0 \\
0 & 1 & 0 \\
0 & 0 & 1
\end{array}\right], \\
& \Gamma_{2}=\left[\begin{array}{ccc}
0.5 & 0 & 0 \\
0 & 0.5 & 0 \\
0 & 0 & 0.5
\end{array}\right] \text {, } \\
& \Gamma_{3}=\left[\begin{array}{cccc}
0.2 & 0 & 0 & 0 \\
0 & 0.2 & 0 & 0 \\
0 & 0 & 0.2 & 0 \\
0 & 0 & 0 & 0.2
\end{array}\right] \text {, } \\
& \Gamma_{4}=\left[\begin{array}{cccc}
0.5 & 0 & 0 & 0 \\
0 & 0.5 & 0 & 0 \\
0 & 0 & 0.5 & 0 \\
0 & 0 & 0 & 0.5
\end{array}\right] \text {, } \\
& M=\left[\begin{array}{ccc}
1+\sin t & 2 & -\cos 2 t \\
-1 & 3+\sin 3 t & 0.5 \\
3 \cos 2 t & -3 \cos 3 t & 2 \sin t \\
1 & -2 & 3 \sin 3 t
\end{array}\right] \text {, } \\
& \sigma_{i}\left(t, e_{i}(t), 1\right)=\operatorname{diag}\left\{\left(\frac{\sqrt{2}}{2}\right) e_{i 1}(t),\left(\frac{\sqrt{2}}{2}\right) e_{i 1}(t),\left(\frac{\sqrt{2}}{2}\right) e_{i 1}(t)\right\} \text {, } \\
& \sigma_{i}\left(t, e_{i}(t), 2\right)=\operatorname{diag}\left\{\sqrt{2} e_{i 1}(t), \sqrt{2} e_{i 1}(t), \sqrt{2} e_{i 1}(t)\right\},
\end{aligned}
$$




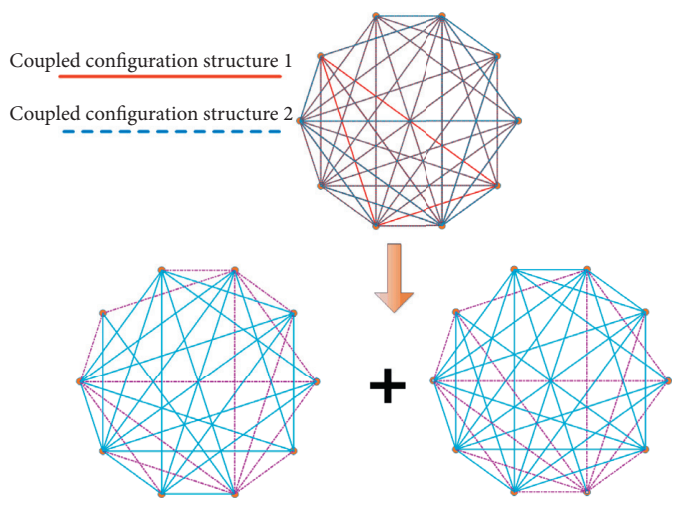

(a)

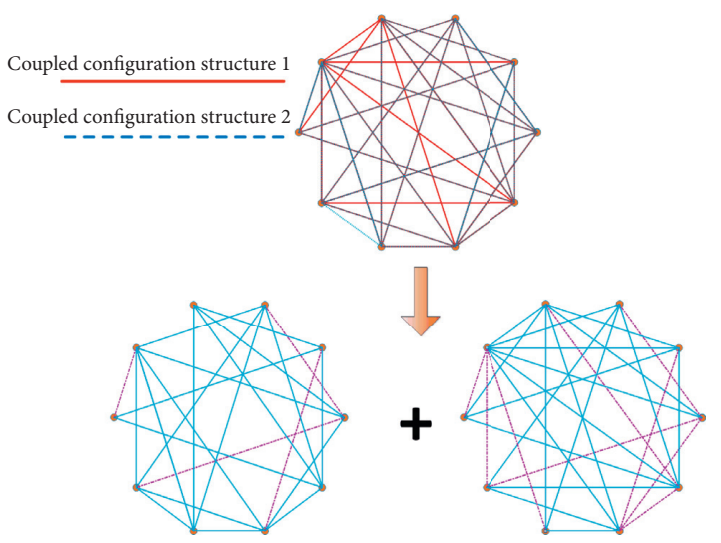

(b)

Figure 10: (a) Topological structure of the driving system in mode 1 and (b) topological structure of the driving system in mode 2.

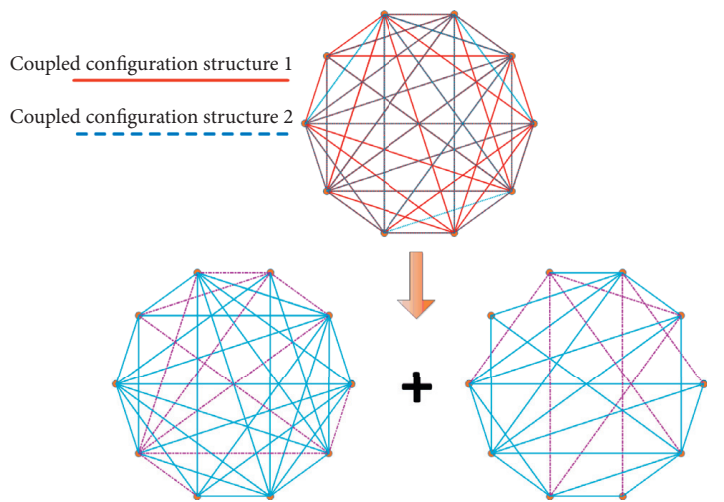

(a)

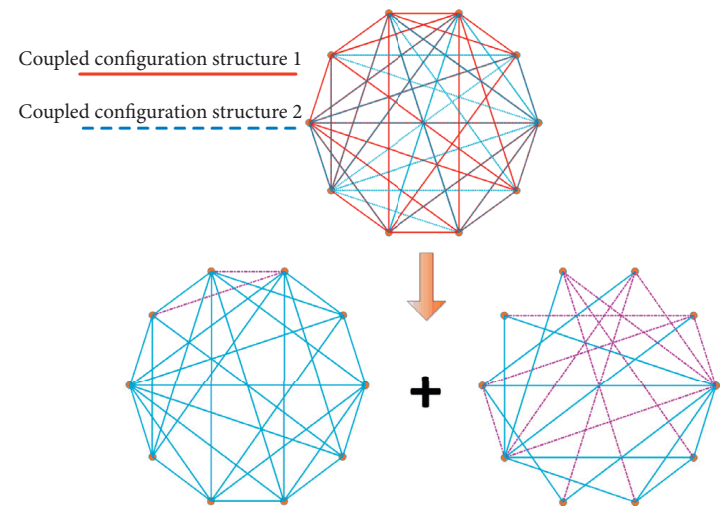

(b)

Figure 11: (a) Topological structure of the response system in mode 1 and (b) topological structure of the response system in mode 2 .

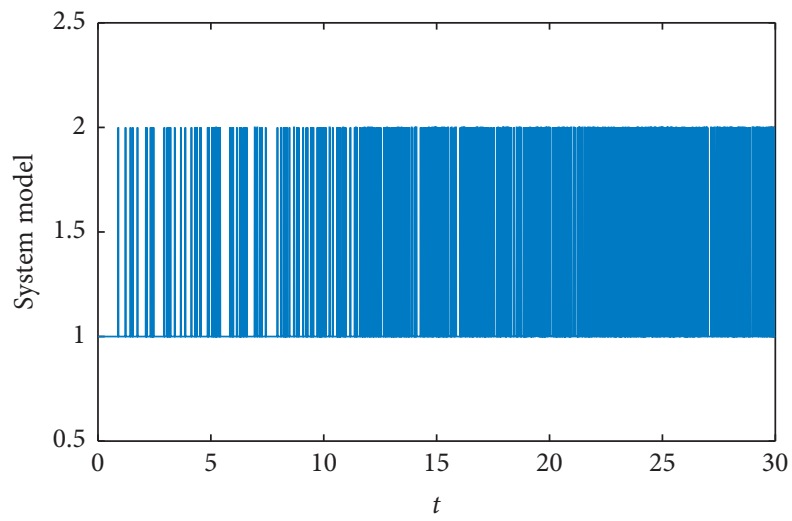

FIGURE 12: Switching of the system mode.

$\tau=0.01, \quad t_{0}=0 s, \quad \psi=15, \quad k_{i}(1)=k_{i}(2)=15, \quad \gamma=0.6, \quad h_{i}(1)=$ $h_{i}(2)=10$, and $s_{i}(1)=s_{i}(2)=12$. Through Lemma 2 and (51), we can obtain $t_{1}=14.427$ by simple calculation.
The time-varying curves of the system synchronization errors $e_{i}(t)(1 \leq i \leq 4)$ are demonstrated in Figure 13 from which one can see that the error converge to zero. Figures 14 


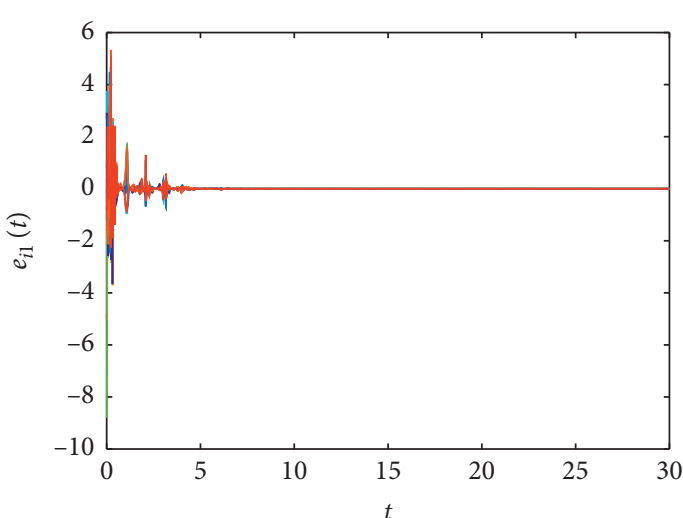

(a)

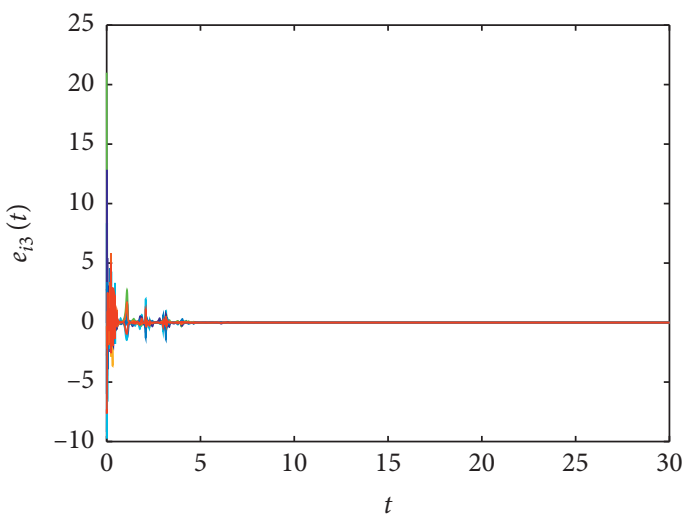

(c)

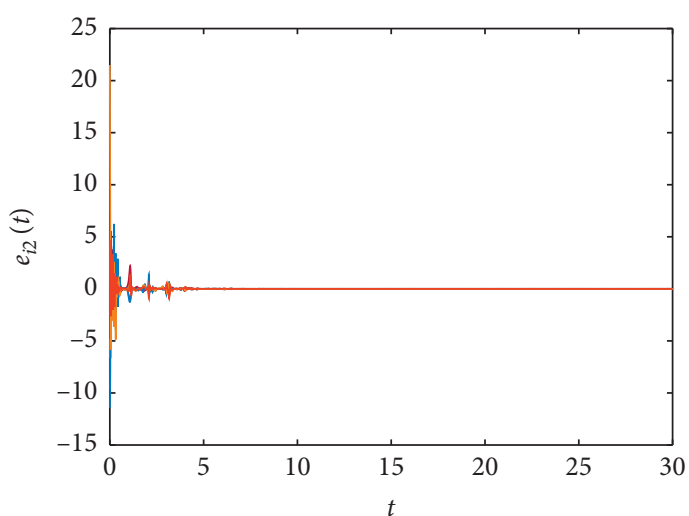

(b)

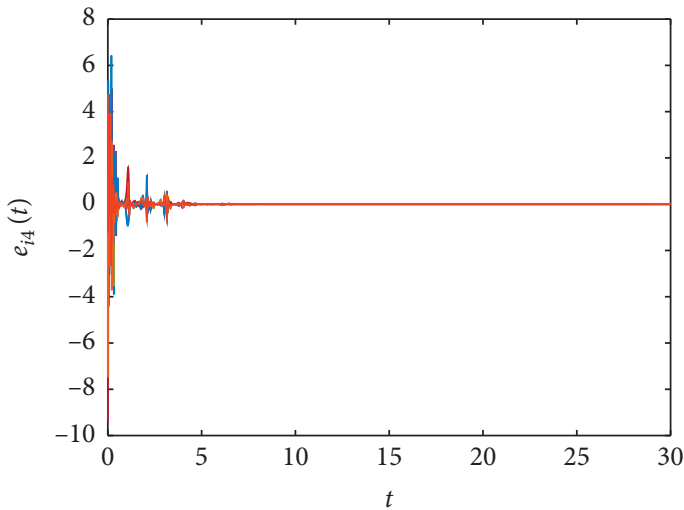

(d)

FIgURE 13: Synchronization errors $e_{i}(t)$ with time evolution.

and 15 give the identification of unknown parameters $\widehat{\alpha}_{i}(i=$ $1,2,3)$ and $\widehat{\beta}_{i}=(i=1,2,3,4)$. It is clear that the estimated parameters are approached to values $(35,3,28)^{T}$ and $(36,3$, $20,1)^{T}$. Figure 16 is the system error values $E(t)$. The conclusion of Figure 16 matches that of Figure 13. The all attest that the control scheme can realize the identification and synchronization of two double-weights Markonian jumping complex networks, effectively.
4.3.2. Simulation Analysis II. In order to further verify that the synchronization criterion can be applied to the synchronization research of double-weights Markovian jumping complex networks, different driving systems are selected for simulation analysis.

The Liu system is taken as the node dynamic of the drive complex network. Among them, $k=1$ and $h=4$ :

$$
\left(\begin{array}{c}
\dot{x}_{i 1}(t) \\
\dot{x}_{i 2}(t) \\
\dot{x}_{i 3}(t)
\end{array}\right)=\left(\begin{array}{c}
0 \\
-k x_{i 1}(t) x_{i 3}(t) \\
h x_{i 1}(t)^{2}
\end{array}\right)+\left(\begin{array}{ccc}
x_{i 2}(t)-x_{i 1}(t) & 0 & 0 \\
0 & x_{i 1}(t) & 0 \\
0 & 0 & -x_{i 3}(t)
\end{array}\right)\left(\begin{array}{c}
\alpha_{i 1} \\
\alpha_{i 2} \\
\alpha_{i 3}
\end{array}\right) .
$$

The identifying value of the parameter vector $\alpha_{i}$ is $\alpha_{i}=\left(\alpha_{i 1}, \alpha_{i 2}, \alpha_{i 3}\right)^{T}=(10,40,2.5)^{T}$.

The four-dimensional hyperchaotic Lüchaotic system will be the node dynamic of the response complex network.

Other relevant parameters are the same as those in Section 4.3.1. $t_{1}=17.806$ is calculated.

The time-varying curves of the system synchronization errors $e_{i}(t)(1 \leq i \leq 4)$ are demonstrated in Figure 17. From that, one can see that the error converge to zero. Figure 18 is the identification of unknown parameters $\widehat{\alpha}_{i}(i=1,2,3)$. It is clear that the estimated parameters are approached to the value $(10,40,2.5)^{T}$.

Under the same research background and system parameters, the control scheme proposed in this paper is compared with the control scheme without finite time to demonstrate the better performance of the proposed control scheme. Figures 19 and 20 are the simulation results under the control scheme without finite time. Figure 19 reveals the 


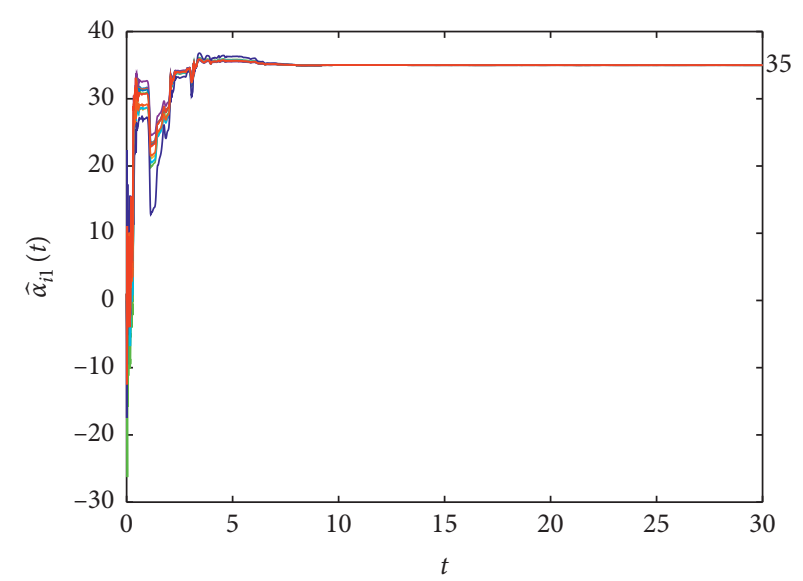

(a)

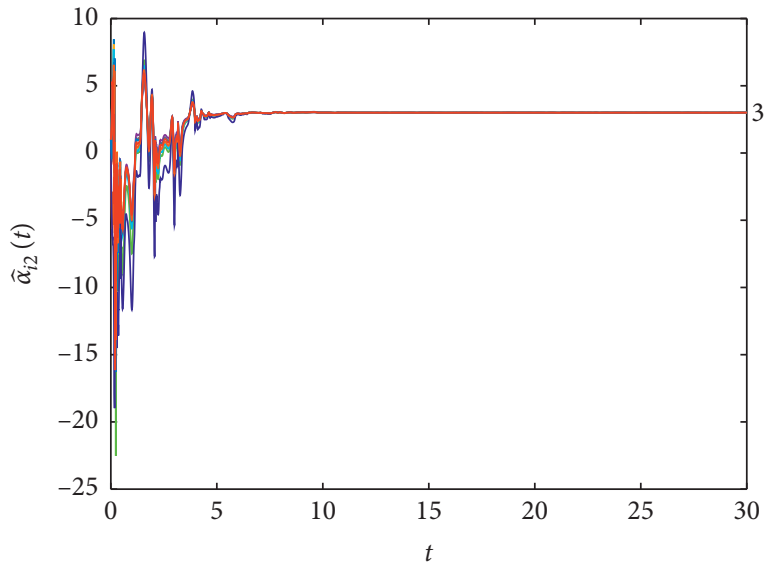

(b)

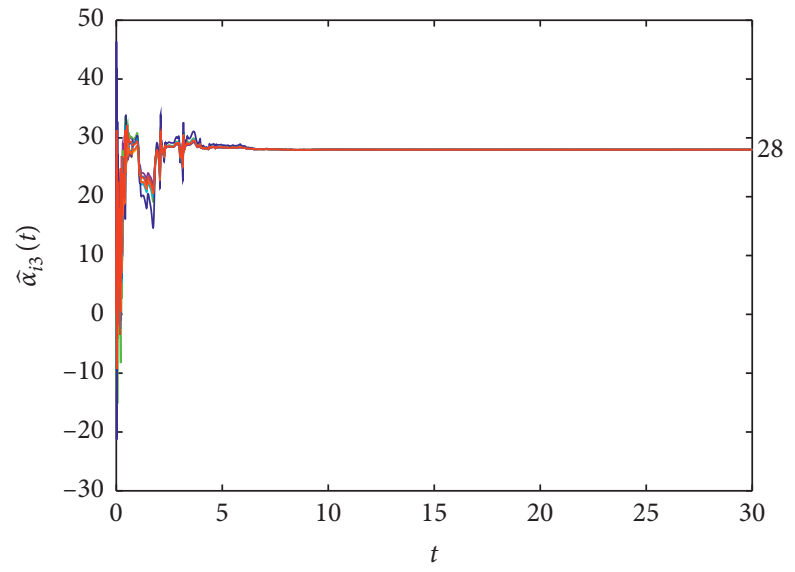

(c)

FIgURE 14: Identification of the uncertain parameters $\widehat{\alpha}_{i}(t)$.

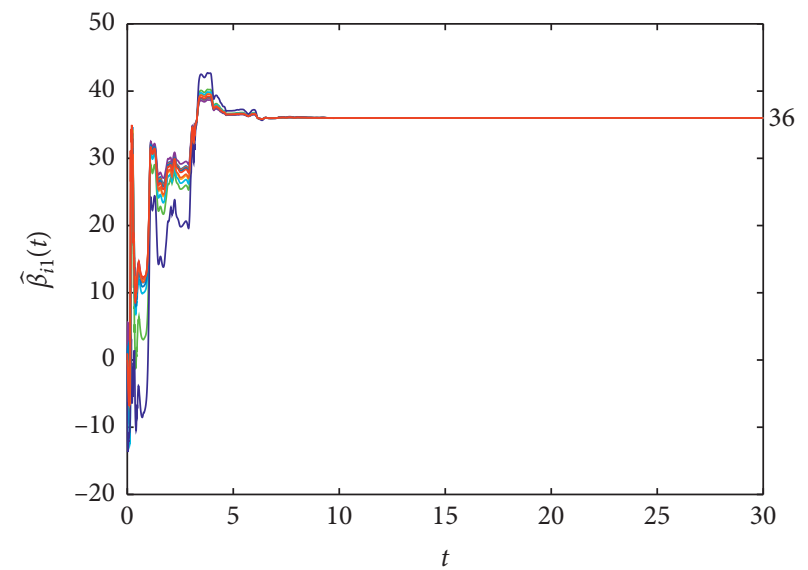

(a)

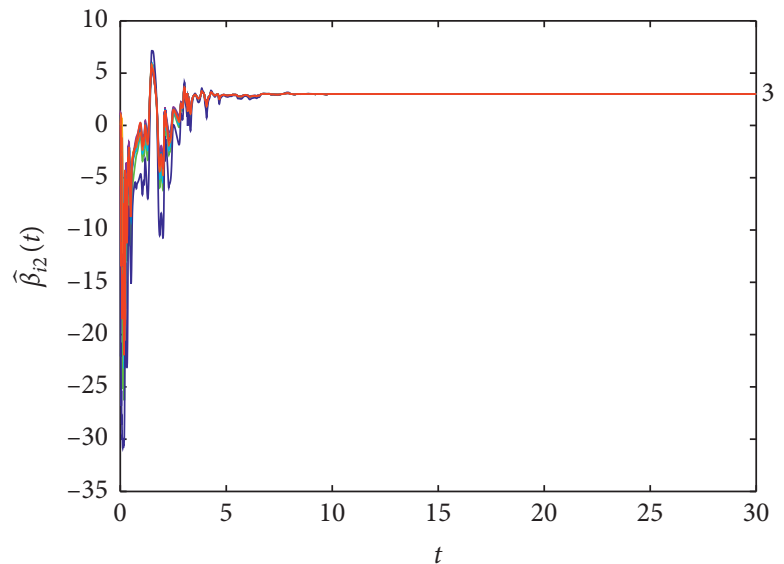

(b)

Figure 15: Continued. 


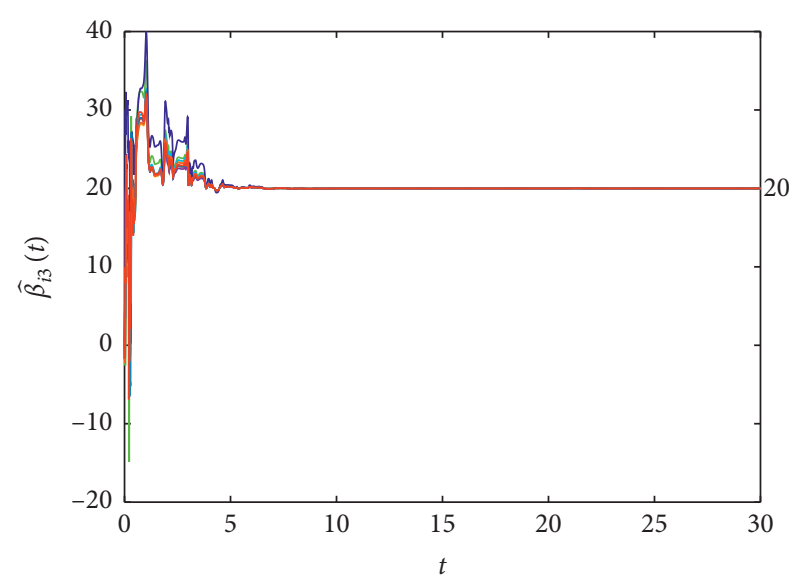

(c)

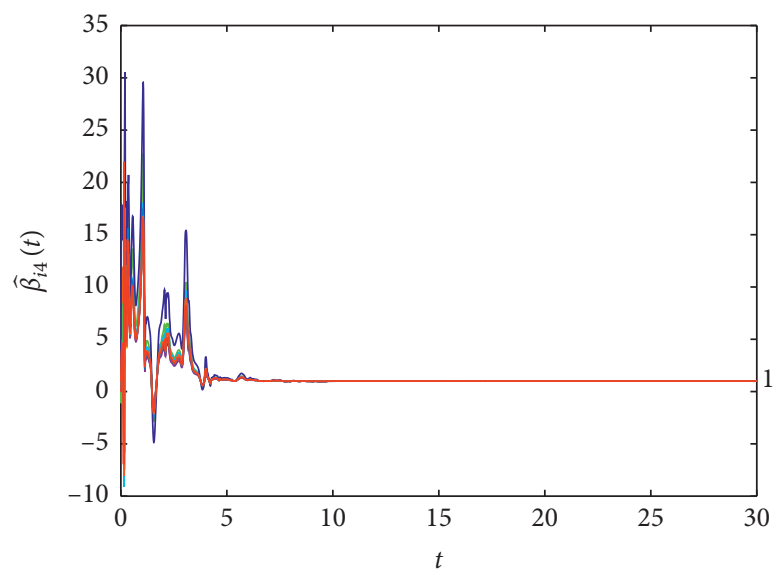

(d)

FIGURE 15: Identification of the uncertain parameters $\widehat{\beta}_{i}(t)$.

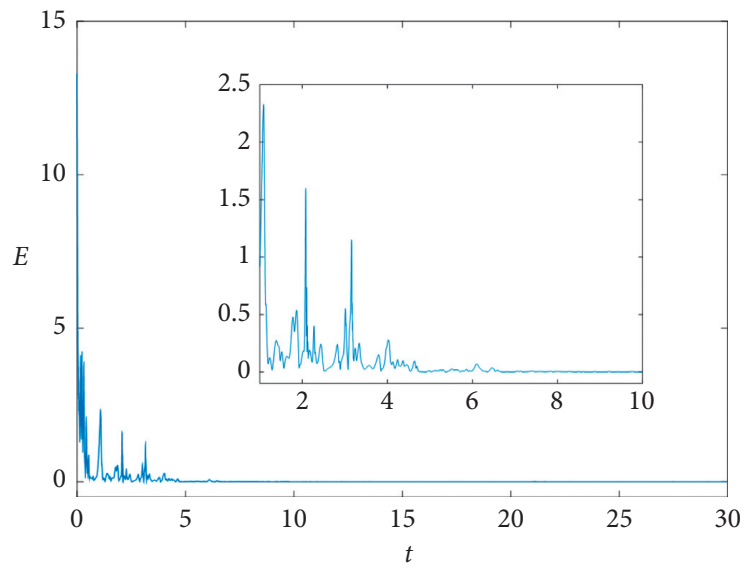

FIgURE 16: Synchronization error value $E(t)$ with varying time.

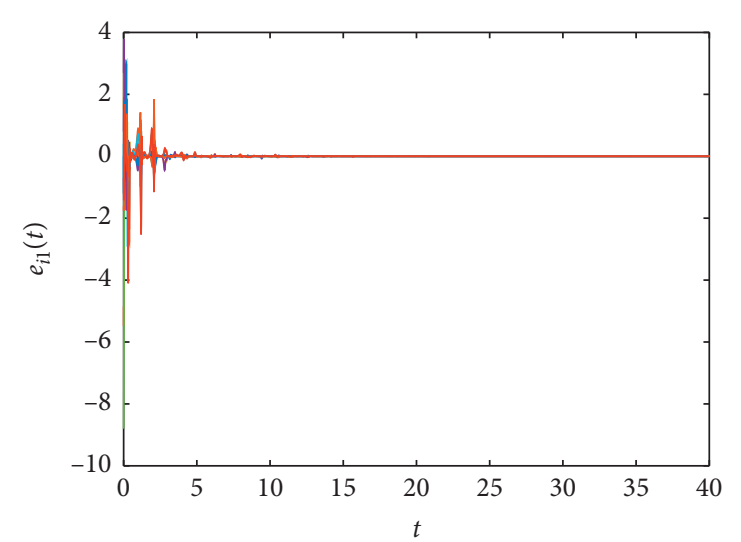

(a)

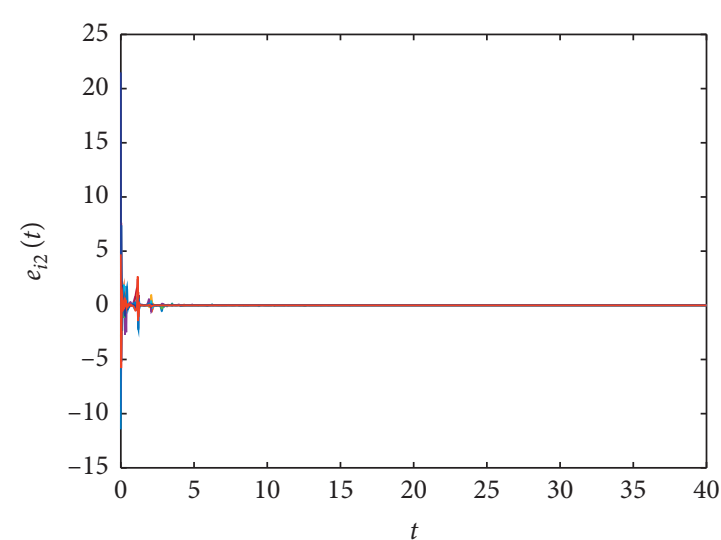

(b)

Figure 17: Continued. 


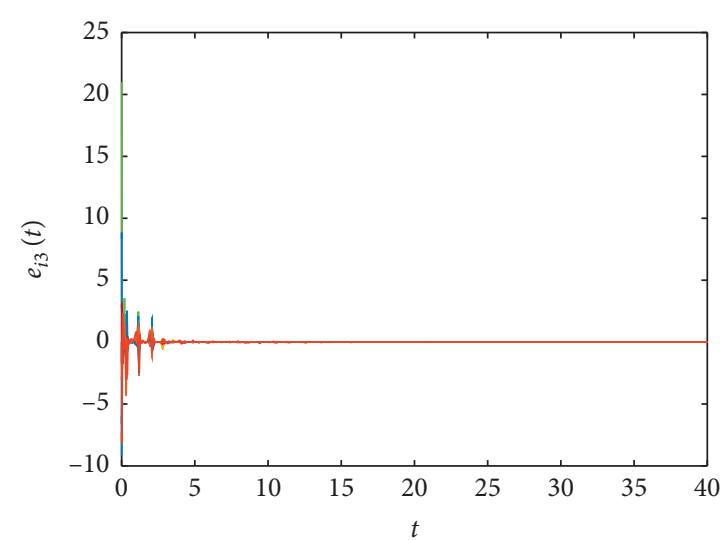

(c)

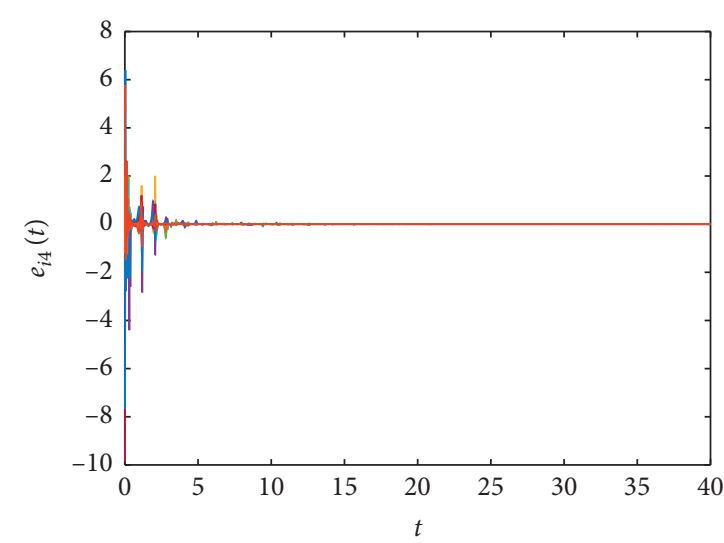

(d)

FIgURE 17: Synchronization errors $e_{i}(t)$ with time evolution.

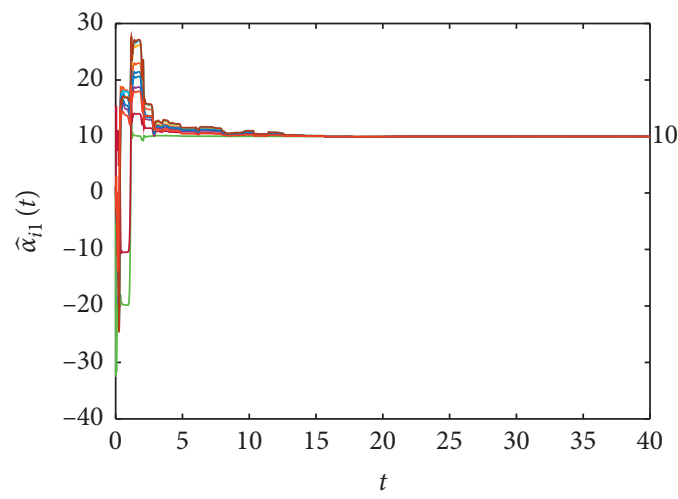

(a)

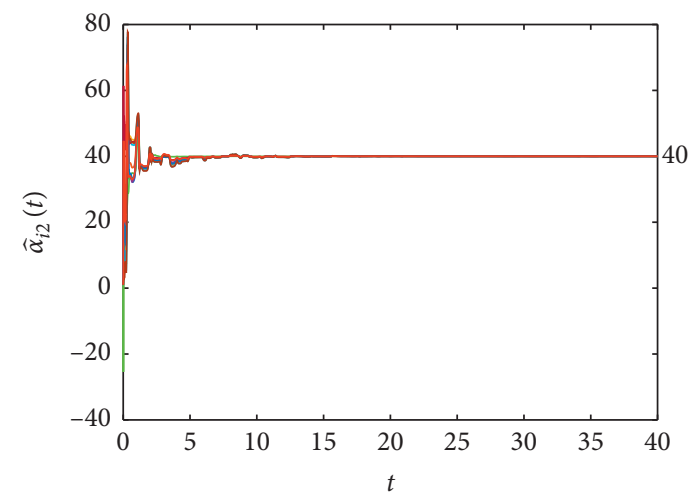

(b)

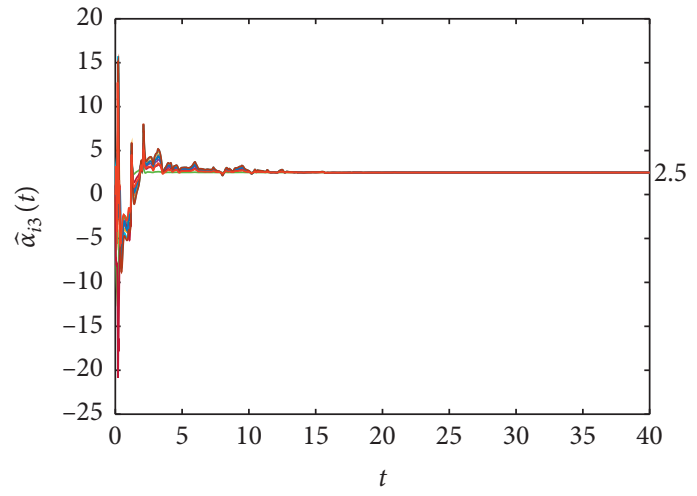

(c)

FIgURE 18: Identification of the uncertain parameters $\widehat{\alpha}_{i}(t)$.

time evolution curves of synchronization error $e_{i}(t)$. Figure 20 indicates that estimated parameter $\widehat{\alpha}_{i}$ converge to their truth values $(10,40,2.5)^{T}$. By comparing with the proposed control scheme in Figures 17 and 18, it is obvious that the proposed control scheme can achieve synchronization and identification more effectively and cost less time. Figure 21 is the synchronization error value $E(t)$ with time-varying under the control scheme with finite time and without finite time.

These illustrate that the synchronization and identification of the drive and response networks have been effectively achieved in finite time. 


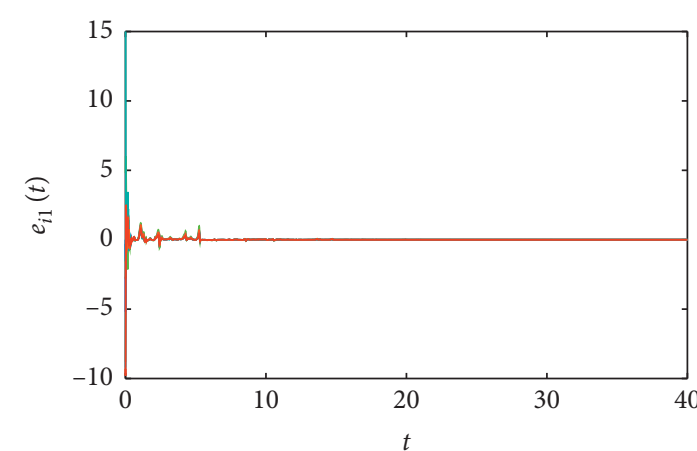

(a)

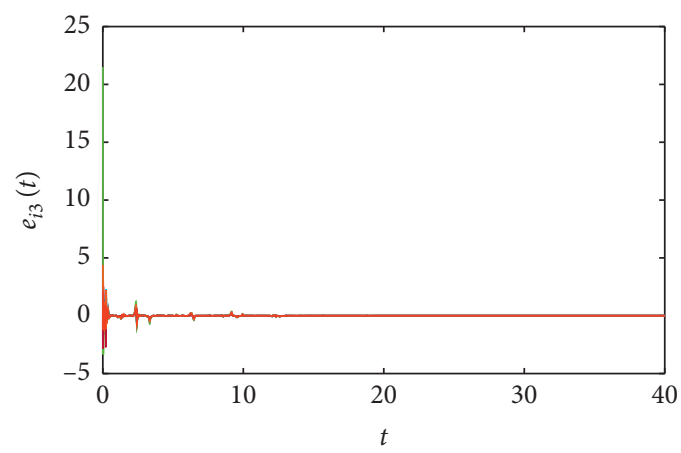

(c)

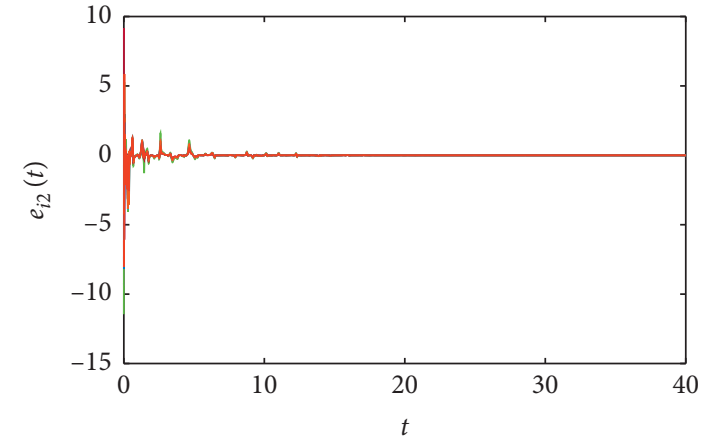

(b)

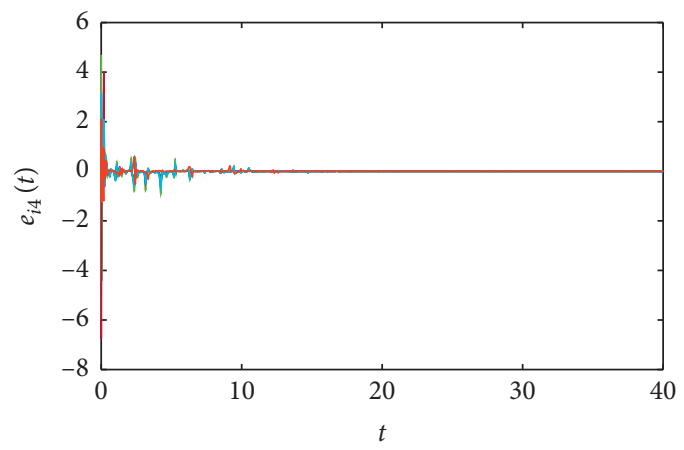

(d)

FIGURE 19: Synchronization errors $e_{i}(t)$ with time evolution under the control scheme without finite time.

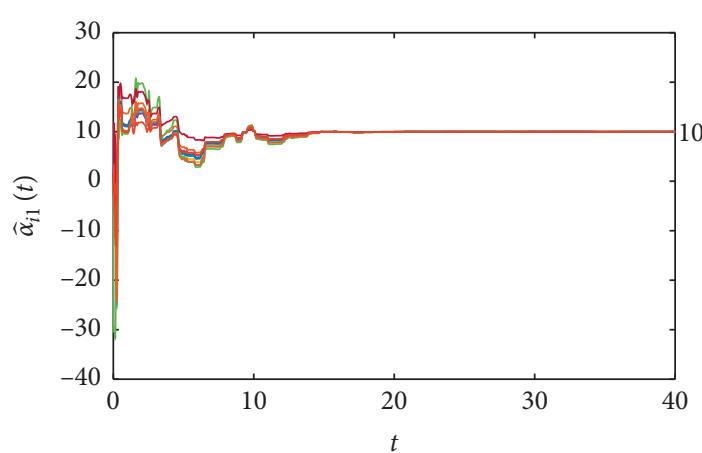

(a)

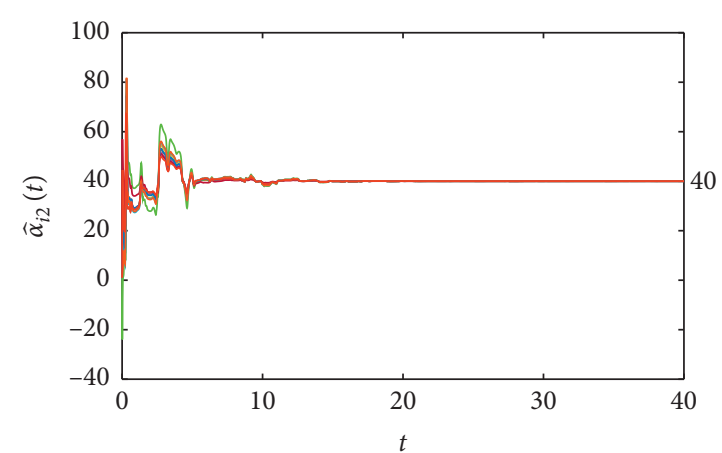

(b)

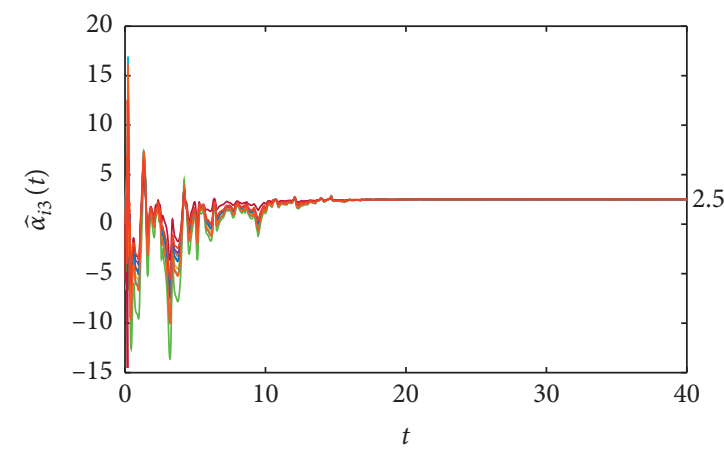

(c)

FIGURE 20: Identification of the uncertain parameter $\widehat{\alpha}_{i}(t)$ under the control scheme without finite time. 


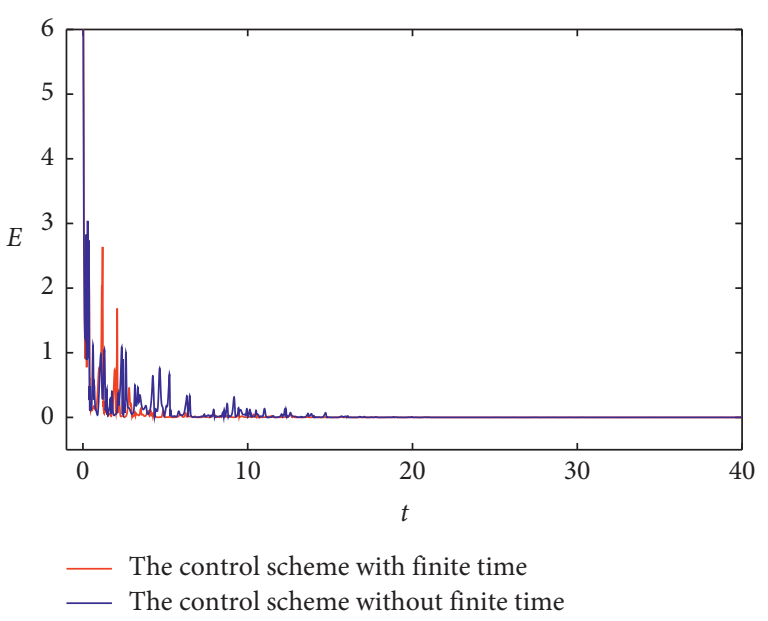

Figure 21: Synchronization error value $E(t)$ with time-varying under the control scheme with finite time and without finite time.

\section{Conclusions}

In this paper, the models of nonidentical dimension Markovian jumping complex networks with stochastic perturbations under the conditions of single weight and double weights are established, firstly. Then, by means of Itô's formula and finite-time convergence theory, feedback controllers and some update laws are given under corresponding conditions to realize the synchronization, and the value of uncertain parameters in networks can be identified successfully. Finally, the simulation results are shown and the effectiveness and feasibility of the proposed method and the method can be applied to various complex dynamic networks.

\section{Data Availability}

No data were used to support this study.

\section{Conflicts of Interest}

The authors declare that they have no conflicts of interest.

\section{Acknowledgments}

This work was supported by the National Natural Science Foundation of Shaanxi Province (Grant no. 2019JQ-726), Scientific Research Program Funded by Shaanxi Provincial Education Department (Grant no. 18JK0558), and Key Research and Development Program of Shaanxi Province (Grant no. 2018ZDXM-GY-169).

\section{References}

[1] F. Sun, H. Peng, J. Xiao, and Y. Yang, "Identifying topology of synchronous networks by analyzing their transient processes," Nonlinear Dynamics, vol. 67, no. 2, pp. 1457-1466, 2012.

[2] G. Cai, Q. Yao, and H. Shao, "Global synchronization of weighted cellular neural network with time-varying coupling delays," Communications in Nonlinear Science and Numerical Simulation, vol. 17, no. 10, pp. 3843-3847, 2012.
[3] W. W. Wang and J. D. Cao, "Synchronization in an array of linearly coupled networks with time-varying delay," Physica A: Statistical Mechanics and its Applications, vol. 366, pp. 197-211, 2006.

[4] Y. Xu, W. Zhou, and J. Fang, "Topology identification of the modified complex dynamical network with non-delayed and delayed coupling," Nonlinear Dynamics, vol. 68, no. 1-2, pp. 195-205, 2012.

[5] T. Nishikawa, A. E. Motter, Y. C. Lai, and F. C. Hoppensteadt, "Heterogeneity in oscillator networks: are smaller worlds easier to synchronize?" Physical Review Letters, vol. 91, no. 1, Article ID 014101, 2003.

[6] J. Wang, H. Zhang, Z. Wang, and Q. Shan, "Local synchronization criteria of markovian nonlinearly coupled neural networks with uncertain and partially unknown transition rates," IEEE Transactions on Systems, Man, and Cybernetics: Systems, vol. 47, no. 8, pp. 1953-1964, 2017.

[7] J.-Y. Wang, H.-G. Zhang, Z.-S. Wang, and H.-G. Liang, "Cluster exponential synchronization of a class of complex networks with hybrid coupling and time-varying delay," Chinese Physics B, vol. 22, no. 9, Article ID 090504, 2013.

[8] Y. Wang and J. Cao, "Cluster synchronization in nonlinearly coupled delayed networks of non-identical dynamic systems," Nonlinear Analysis Real World Applications, vol. 14, no. 1, pp. 842-851, 2012.

[9] Y. R. Liu, Z. D. Wang, J. L. Liang, and X. H. Liu, "Synchronization of coupled neutral-type neural networks with jumping-mode-dependent discrete and unbounded distributed delays," IEEE Trans on Cybernetics, vol. 43, no. 1, pp. 102-114, 2013.

[10] X. Hao and J. Li, "Stochastic synchronization for complex dynamical networks with time-varying couplings," Nonlinear Dynamics, vol. 80, no. 3, pp. 1357-1363, 2015.

[11] L. I. Wang, M. A. Zhicai, and S. Hongjun, "Finite-time stochastic generalized outer synchronization of time-delayed complex dynamical networks," Journal of Shandong University(Engineering Science), vol. 47, no. 3, pp. 1-8, 2017.

[12] L. L. Zhang, Y. H. Wang, and Q. R. Wang, "Asymptotic synchronization for nonlinear coupled complex dynamical networks with different-dimension nonlinear nodes," Control and Decision, vol. 29, pp. 537-540, 2014.

[13] Q. Song and Z. Zhao, "Cluster, local and complete synchronization in coupled neural networks with mixed delays and nonlinear coupling," Neural Computing and Applications, vol. 24, no. 5, pp. 1101-1113, 2014.

[14] L. Li, W. C. Ho Daniel, J. Cao, and J. Lu, "Pinning cluster synchronization in an array of coupled neural networks under event-based mechanism," Neural Networks, vol. 76, pp. 1-12, 2016.

[15] Y. G. Jin, S. M. Zhong, and N. An, "Function projective synchronization between two different complex networks with correlated random disturbances," Chinese Physics B, vol. 24, no. 4, Article ID 049202, 2015.

[16] W. Wu, D. Lai, and H. Lu, "Generalized synchronization of the fractional-order chaos in weighted complex dynamical networks with nonidentical nodes," Nonlinear Dynamics, vol. 63, no. 1-2, pp. 667-679, 2011.

[17] X. Huang and Y. Ma, "Finite-time $H_{\infty}$ sampled-data synchronization for Markovian jump complex networks with time-varying delays," Neurocomputing, vol. 296, pp. 82-99, 2018.

[18] J. L. Wang, H. N. Wu, T. Huang, S. Y. Ren, and J. Wu, "Passivity and output synchronization of complex dynamical networks with fixed and adaptive coupling strength," IEEE 
Transactions on Neural Networks and Learning Systems, vol. 29, no. 2, pp. 364-376, 2018.

[19] C. Li, W. Sun, and J. Kurths, "Synchronization between two coupled complex networks," Physical Review E, vol. 76, no. 4, p. $1,2007$.

[20] M. Zhao, H. G. Zhang, Z. L. Wang, and H. J. Liang, "Observerbased lag synchronization between two different complex networks," Communications in Nonlinear Science and $\mathrm{Nu}$ merical Simulation, vol. 19, no. 6, pp. 2048-2059, 2014.

[21] X. Wu and $\mathrm{H}$. Lu, "Outer synchronization of uncertain general complex delayed networks with adaptive coupling," Neurocomputing, vol. 82, pp. 157-166, 2012.

[22] Q. Xie, C. H. Mu, Y. Li, G. Wu, and R. Jia, "Finite-time projective lag synchronization and Identification between two different Markovian Jumping complex networks with stochastic perturbation," in Proceedings of the 38th Chinese Control Conference (CCC2019), Guangzhou, China, July 2019.

[23] N. Yang and C. Liu, "A novel fractional-order hyperchaotic system stabilization via fractional sliding-mode control," Nonlinear Dynamics, vol. 74, no. 3, pp. 721-732, 2013. 